\title{
XIII. LE DROIT D'ÉTAT DU SAINT-EMPIRE DANS L'OPINION PUBLIQUE FRANÇAISE: JOURNAUX ET TRACTS - REGARDS CROISÉS
}

\author{
1. Le problème de l'existence d'une opinion publique \\ au XVII siècle et les informations fournies \\ par la »Gazette de France« sur le droit allemand
}

Dans ce livre, nous avons souvent parlé des articles consacrés à l'Allemagne dans la »Gazette de France«, notamment à l'occasion de la conclusion des traités de paix. En effet, non seulement les livres imprimés et les mémorandums politiques manuscrits, mais aussi la presse périodique, et en particulier la »Gazette de France «, renseignaient le lectorat français sur les événements politiques et militaires qui se produisaient en Allemagne et dans l'Empire. Au XVIIe siècle, on trouve au nombre des collaborateurs de la »Gazette « Epstein, originaire d'Allemagne, ainsi que les frères Dupuy, qui faisaient partie des plus grands érudits de leur époque. Au XVIII ${ }^{e}$ siècle, on note parmi les rédacteurs du journal des employés du ministère des Affaires étrangères français et d'excellents spécialistes du droit public du Saint-Empire, comme Pfeffel et Gérard de Rayneval. La »Gazette de France « représente donc une source formidable pour connaître l'interprétation qui prévalait dans les cercles proches du gouvernement français à propos des événements politiques dans le Saint-Empire. Toutefois, malgré les très bonnes connaissances de certains rédacteurs, notamment en matière de droit public allemand, cette presse ne fournissait aux Français que très peu d'informations sur le droit public de l'Empire. Néanmoins, des événements importants, comme l'étaient surtout les traités de paix, les élections impériales, certaines délibérations ou décisions de la diète, retenaient l'intérêt des collaborateurs de la "Gazette«. Cela s'explique certainement par l'objectif même de la presse périodique, qui consistait à informer ses lecteurs des événements qui avaient lieu en Allemagne, et non pas à décrire les structures constitutionnelles de l'Empire. Rappelons cependant que Pfeffel et Rayneval publièrent des ouvrages sur le droit public allemand. Les rédacteurs de la »Gazette « connaissaient donc souvent très bien la Constitution du Saint-Empire, mais un périodique ne leur fournissait pas l'occasion de l'étudier.

Après avoir surtout examiné des articles de la »Gazette «parus au XVII ${ }^{e}$ siècle, voyons quelles informations ce journal donne sur l'Allemagne au milieu du XVIII ${ }^{\mathrm{e}}$ À cet effet, nous avons dépouillé, à titre d'exemples, les numéros de $1738^{1}$, l'année où fut signée la paix définitive entre la France et l'empereur à Vienne, en Autriche. Comme au XVII' siècle, la »Gazette« rend compte des séan-

1 Gazette [de France], ici Recveil de tovtes les Gazettes, novvelles ordinaires \& extraordinaires \& autres relations. Recueil des nouvelles ordinaires et extraordinaires, relations et recits des choses avenues, tant en ce Royaume qu'ailleurs [1738], Paris 1701 [i.e. 1739]. 
ces de la diète de l'Empire. Mais, en 1738, les délibérations de la diète de Ratisbonne sont surtout évoquées dans des contextes militaires, en particulier quand elle accorde des mois romains à l'empereur ${ }^{2}$ ou quand il s'agit de réparer des fortifications ${ }^{3}$ ou bien de subvenir aux coûts de leur entretien et de leur garde ${ }^{4}$. Cependant, ces articles évoquent aussi les griefs de religion et les affaires judiciaires discutés à la diète 5 . Dans les nouvelles en provenance de Vienne, les aspects militaires occupent également une place prédominante, accompagnés des nouvelles de la cour, par exemple des festivités à l'occasion de l'anniversaire de l'empereur $^{6}$. Mais ces nouvelles de Vienne concernent également la vie intellectuelle et culturelle, comme les prix accordés par l'Académie de peinture, de sculpture et $\mathrm{d}^{\text {'architecture }}{ }^{7}$. On s'intéresse particulièrement à la nomination de nouveaux officiers et ministres, voire à celle d'un conseiller aulique (Reichshofrat) ${ }^{8}$. Vienne est aussi le lieu par lequel arrivent à Paris les nouvelles en provenance de la frontière avec l'Empire ottoman ou des Balkans. Quoi qu'il en soit, les articles sur Vienne fourmillent de nouvelles militaires. En revanche, les articles de la $\gg \mathrm{Ga}$ zette « parus en 1738 qui traitent de l'Allemagne ne font qu'une place mineure aux problèmes juridiques et constitutionnels, si ce n'est dans le cadre de certaines informations en provenance de Ratisbonne.

Dans la seconde moitié du XVII e siècle, la »Gazette «s'était pourtant intéressée davantage aux délibérations de la diète de l'Empire, surtout certaines années cruciales, comme en 1684 , quand la diète débattait d'un traité de paix ou de trêve à conclure avec la France; à ce moment-là, la "Gazette « faisait régulièrement des comptes rendus assez précis des séances des collèges 9 . De toute évidence, l'intérêt que les rédacteurs du journal accordaient à la diète de l'Empire diminua sensiblement au cours du demi-siècle suivant.

Cependant, la lecture de certains tracts du XVIII' siècle peut permettre d'approfondir cette analyse des journaux et des brochures, qui constitue un aspect

${ }^{2}$ Cf., par exemple, ibid., $\mathrm{n}^{\circ} 8$ (22 février 1738 ), p. 85-96, ici p. 86-87, l'article »De Ratisbonne, le 4 Fevrier $1738 \ll$.

${ }^{3}$ Ibid., $n^{\circ} 9$ (1 ${ }^{\text {er }}$ mars 1738), p. 97-108, ici p. 98-99, l'article »De Ratisbonne, le 11 Fevrier $1738 \ll$.

4 Ibid., no 15 (5 avril 1738), p. 161-165, ici p. 164-165, l'article »De Ratisbonne, le 17 Mars $1738 \ll$.

5 Ibid., no 17 (19 avril 1738), p. 185-196, ici p. 189, l'article »De Ratisbonne, le 31 Mars $1738 \ll$.

6 Ibid., $n^{\circ} 44$ (25 octobre 1738), p. 507-518, ici p. 509-510, l'article »De Vienne, le 5 Octobre 1738 « (l'anniversaire de Charles VI était le 1er octobre).

7 Ibid., no 50 (6 décembre 1738), p. 579-590, ici p. 584-585, l'article »De Vienne, le 15 Novembre 1738 «. En ce qui concerne la vie intellectuelle et culturelle de l'Allemagne, la »Gazette« observe en général attentivement ce qui se passe à Dresde.

8 Ibid., no 22 (24 mai 1738), p. 245-256, ici p. 247-249, l'article »De Vienne, le 3 May $1738 \ll$.

${ }^{9}$ Cf. Gazette de [France], ici Recveil de tovtes les Gazettes, novvelles ordinaires \& extraordinaires \& autres relations. Recvëil des novvelles ordinaires et extraordinaires, relations et recits des choses avenues tant en ce Royaume qu'ailleurs, pendant l'année mil six cens quatre-vingts quatre, Paris 1685. 
important de l'étude de l'opinion publique ${ }^{10}$. D'ailleurs, comme nous avons déjà pu l'observer, ce type de publications pouvait aussi exercer une influence sur la politique étrangère. La question de savoir si une opinion publique existait au XVIIe siècle a fait couler beaucoup d'encre. Nous sommes mieux renseignés quant au XVIII', bien connu à cet égard depuis Habermas et son concept de la »bürgerliche Öffentlichkeit “" ${ }^{\prime}$. En ce qui concerne le XVII ${ }^{e}$ siècle, pour lequel le problème est beaucoup plus difficile à résoudre, notons que Georges Livet parle déjà à propos de la première moitié de ce siècle d'un "esprit public «, en analysant les pamphlets, les chansons, les journaux et l'enseignement de l'époque ${ }^{12}$. Si cette problématique extrêmement complexe ne peut pas être étudiée ici de façon très détaillée, il paraît important de noter que ce ne sont pas seulement les écrits des spécialistes qui propageaient des connaissances sur le système politique de l'Allemagne, mais aussi, quoique dans une moindre mesure, les périodiques, les brochures ainsi que des ouvrages littéraires et philosophiques, tels que les »Mémoires « de Saint-Simon (qui en parle occasionnellement) et les écrits de Montesquieu (qui prête une attention particulière à l'organisation politique du Saint-Empire).

\section{La cour et l'éducation des princes}

Saint-Simon, pour sa part, regrettait l'obscurité et la rareté des nouvelles en provenance d'Allemagne ${ }^{13}$. Néanmoins, il était informé des principaux événements dynastiques, politiques et juridiques, tels que la mort de Léopold Ier 14 et la mise au ban de l'Empire des princes électeurs de Bavière et de Cologne. Il évoque également les prétentions de préséance de l'électeur de Bavière, la création de princes de l'Empire, la conclusion des traités de paix entre la France et l'Empire, la titulature employée dans les lettres échangées entre le roi de France et l'empereur, la faiblesse de l'autorité impériale dans les États de Toscane, etc. De plus, Saint-Simon, qui, outre le latin, prétend avoir su parler "parfaitement l'allemand « depuis sa jeunesse, mentionne certains écrivains et historiens de son temps, tels qu'Antoine Varillas, que nous avons déjà rencontré comme auteur ayant écrit sur l'Alle-

10 Pour les aspects méthodiques du problème, surtout dans une perspective franco-allemande, cf. maintenant WREDE, Das Reich und seine Feinde (avec la bibliographie des ouvrages plus anciens).

11 Cf. Jürgen Habermas, Strukturwandel der Öffentlichkeit. Untersuchungen zu einer Kategorie der bürgerlichen Gesellschaft, Neuwied 1962 (Politica, 4).

12 Georges LIVET, La guerre de Trente Ans, Paris 61994 [première édition: 1963], p. 100.

13 Cf. SaINT-Simon, Mémoires, ici t. II, p. 344-363. Rappelons que la plus grande partie des „Mémoires « du duc de Saint-Simon (1675-1755) recouvrant la période de sa vie qu'il passa à la cour (1691-1723) a été rédigée entre 1739 et 1749. Pour Saint-Simon et l'Allemagne, cf. Jürgen Voss, Der Herzog von Saint-Simon und Deutschland, dans: DuchHARDT, SCHMIDT (dir.), Deutschland und Frankreich in der frühen Neuzeit, p. 439-465.

14 Cf. Saint-Simon, Mémoires, t. II, p. 462-477. 
magne ${ }^{15}$. Saint-Simon brosse également un portrait très positif de François de Callières, qu'il considérait comme un homme et un négociateur honnête, caractérisé par l'amour de l'État et le courage de dire la vérité au roi et aux ministres, quitte à leur déplaire; il avouait recevoir de lui »une grande instruction «16. Le duc de Saint-Simon connaissait le Saint-Empire à travers ses voyages, et nous a laissé une description touchante de la destruction du Palatinat par Louvois:

Nous passâmes à Spire [écrit-il à propos de l'année 1694] dont je ne pus m'empêcher de déplorer la désolation. C'étoit une des plus belles et des plus florissantes villes de l'Empire; elle en conservoit les archives, elle étoit le siège de la Chambre impériale, et les diètes de l'Empire s'y sont souvent assemblées. Tout y étoit renversé par le feu que $M$. de Louvois y avoit fait mettre ainsi qu'à tout le Palatinat, au commencement de la guerre, et ce qu'il y avoit d'habitants, en très-petit nombre, étoient huttés sous ces ruines ou demeurant dans les caves ${ }^{17}$.

Le fait que la ville de Spire était le siège des plus prestigieuses institutions impériales était donc bien présent à l'esprit du duc quand il y passa. Ce personnage de la cour observait attentivement les débats autour de la création d'une neuvième dignité électorale par l'empereur, qui avait »causé tant de mouvements dans l'Empire et en Europe, comme il le notait en 1699 et en $1700^{18}$. Pour Saint-Simon, Léopold ler fut le monarque allemand le plus puissant depuis Charles Quint; en effet, il dit de lui au moment de sa mort: »Une laideur ignoble, une mine basse, une simplicité fort éloignée de la pompe impériale, ne l'empêcha pas d'en pousser l'autorité beaucoup plus loin qu'aucun de ses prédécesseurs, si on en excepte Charles V, et sa vie extérieure, plus monacale que ce prince, ne l'empêcha pas de se servir de toutes sortes de voies pour arriver à ses fins«. Le duc lui reprochait en particulier »ses usurpations [...] contre le serment de ses capitulations «. Pour Saint-Simon, le ban impérial infligé aux électeurs de Cologne et de Bavière était une énième preuve du fait que »l'Allemagne étoit subjuguée depuis Charles $\mathrm{V}_{\text {« }}$ par les empereurs de la maison d'Autriche. Selon lui, ces derniers n'avaient point perdu leur autorité 19 .

À certains égards, Saint-Simon croit légitime une comparaison entre les institutions allemandes et françaises. À titre d'exemple, il soutient que, au Moyen Âge, le rôle politique des pairs de France comme »colonnes de l'État« pouvait être comparé à celui des électeurs dans l'Empire, abstraction faite du droit de vote dans la monarchie élective qu'était l'Empire, à la différence du royaume de France ${ }^{20}$. Certains problèmes constitutionnels cruciaux ne sont esquissés que de façon très passagère, comme l'opposition des princes protestants de l'Empire au quatrième article de la paix de Ryswick ${ }^{21}$.

15 Cf. ibid., t. I, p. 176, 304 et 626-627; t. II, p. 604-617; t. III, p. 310-332; t. IV, p. 7-34 et 413-428; t. V, p. 1125-1144.

16 Cf. ibid., t. I, p. 352-354; t. II, p. 522 (citation); t. V, p. 471.

17 Cf. ibid., t. I, p. 179-180.

18 Cf. ibid., p. 608 (citation) et 811.

19 Ibid., t. II, p. $474-475$ et 604.

20 Cf. ibid., t. IV, p. $477-480$ (citation p. 480 ).

21 Cf. ibid., t. I, p. 423-425. 
Néanmoins, il est évident que le nombre des personnes qui s'intéressaient à la Constitution allemande ne se limitait pas à la seule catégorie des professionnels (diplomates, historiens et juristes). Toutefois, il faut supposer que le public cultivé - la cour, la ville, les érudits (séculiers et ecclésiastiques) et les nobles provinciaux - ne disposait que de notions approximatives en matière de droit public allemand. Malgré cette réserve, les notes de lecture conservées dans les manuscrits de plusieurs bibliothèques et les ex-libris de certains manuscrits ou d'ouvrages imprimés que nous avons mentionnés dans notre livre montrent que la lecture de textes sur la Constitution du Saint-Empire n'était pas uniquement l'affaire de quelques spécialistes. C'est la raison pour laquelle nous indiquerons ces $e x$-libris dans notre bibliographie comme un indice de la réception de ces ouvrages. Ajoutons à cela que les connaissances que nous avons de la composition de certaines bibliothèques nobiliaires montrent que des livres sur l'Allemagne, son histoire, son droit, sa géographie et la généalogie de ses dynasties ne manquaient pas dans les bibliothèques les plus importantes 22 . Outre les documents $d$ 'archives que nous avons déjà cités, les inventaires de certaines bibliothèques privées ainsi que d'autres documents bibliographiques attestent la réception en France des livres imprimés et des manuscrits sur l'Allemagne ${ }^{23}$.

D'ailleurs, l'histoire et la Constitution du Saint-Empire avaient leur place dans l'éducation des princes. En 1727, Élie Christophe Lautz composa un traité ou »Abrégé du droit public de l'Empire«, et ceci »par ordre de Monseigneur le duc de Noailles $\ll^{24}$. Ce traité devait servir à l'éducation des enfants du duc ${ }^{25}$. Élie

22 On peut par exemple mentionner le fait que, dans la bibliothèque de Gaston d'Orléans, qui était un homme cultivé au goût prononcé pour les livres d'histoire, trois atlas sur dix-huit concernaient la géographie allemande, cf. Christian BOUYER, Gaston d'Orléans (16081660). Séducteur, frondeur et mécène, Paris 1999, p. 282.

23 Cf., par exemple, archives départementales du Nord, Lille, B.19891, titre d'après l'inventaire: »Bibliographie. Notes et fragments (XVII ${ }^{e}-X V I I I^{e}$ s.) «, ici les documents suivants: une liste de deux feuillets intitulée "Livres à vendre avec les prix (parus entre 1571 et 1734), où l'on trouve l'ouvrage de Pufendorf: »Severini de Monzambano de Statu Imperii Germanici. in 12. Genève, 1667 « au prix d'une livre dix sous. Un inventaire de manuscrits mentionne plusieurs pièces concernant l'Allemagne, entre autres un mémoire sur la question de savoir »comment l'Empire fut transféré des mains des François aux princes Saxons en Allemagne«. On retrouve également les traces des éditions de traités internationaux. Enfin, dans la même liasse, on trouve un "Catalogue des livres de monsieur Caesar d'Hennin baron de Boelbecq, trouvez dans la chambre du révérend Père Ambroise Deffontaines " donation de 1690); cette liste contient les écrits de plusieurs juristes allemands, entre autres de Matthias Wesenbeck.

24 »Abrégé du droit public de l'Empire, composé par ordre de Monseigneur le duc de Noailles. Pour l'usage de messieurs ses enfans les comte d'Ayen et marquis de Mouchy, par Elie Christophle Lautz, bibliothécaire de mondit Seigneur, à Paris. 1727«, copie: AE, MD All. 66 fol. 2-102' (titre selon le frontispice, fol. 2; l'inventaire indique comme auteur: Lantz).

25 Pour le rôle de l'histoire dans l'éducation des princes au XVIIIe siècle, cf. Chantal GrELL, L'éducation des princes et l'héritage du passé, dans: FuMAROLI, Grell (dir.), Historiographie de la France, p. 261-304. Cf. également Guido CANZIANI, »Politiques« pour le prince. Traités et manuels au début du règne de Louis XIV, dans: Henri MÉCHOULAN, Joël CoRNETTE (dir.), L'État classique (1652-1715). Regards sur la pensée politique de la France dans le second XVII siècle, Paris 1996, p. 93-111. Plusieurs bibliothèques possèdent aujourd'hui des 
Christophe Lautz était juriste. Nos connaissances sur sa biographie sont lacunaires. Toutefois, nous savons qu'il rédigea un discours pour le jour de l'an 1742, qui fut prononcé à l'hôtel de ville de Strasbourg le 30 décembre $1741^{26}$. En ce qui concerne la forme du gouvernement du Saint-Empire, Lautz adhère à l'école du status mixtus, selon laquelle des éléments monarchiques et des éléments aristocratiques sont mélangés dans sa Constitution. Selon Lautz, il y a, dans l'Empire, un certain équilibre entre ces différents composants. Après avoir parlé de la puissance des empereurs issus des maisons de Charlemagne et des Othons, l'auteur constate une certaine décadence du pouvoir impérial chez les empereurs suivants, qui a été figée par les lois fondamentales, puisque ces empereurs succédant aux Othons »n'ayant pas été assés forts pour rétablir leur ancienne puissance, les princes se maintinrent dans leurs droits, quoyque uniquement fondés sur la coutume et sur la possession, jusqu'à ce que leurs privilèges furent enfin confirmés par des loix publiques nommées communément loix fondamentales de l'Empire ${ }^{27}$. Cependant, cet auteur insiste sur le fait que les états, malgré une large autonomie dans le règlement des affaires intérieures, dépendent toujours de l'empereur et de l'Empire ${ }^{28}$. C'est cette vision modérée d'un empire conciliant monarchie et aristocratie qui fut donc, à en juger par ce manuscrit, enseignée, à la fin des années 1720, aux enfants du duc de Noailles. L'image du Saint-Empire ne s'est pas encore profondément modifiée en France par rapport à celle du XVIIe siècle. L'impact de la tradition sur cette vision de l'Empire est encore attesté par le début du chapitre premier de cet »Abrégé« historique, car Lautz y reprend la théorie de la translatio Imperii et celle des quatre monarchies selon les prophéties de Daniel, puisque l'auteur annonce son dessein de rapporter d'abord, en quelques mots, »'histoire de l'origine de l'Empire, et comment il est passé à la nation germanique ${ }^{29}$. Puis il résume très brièvement l'histoire de Rome avant l'avènement de Constantin, en expliquant de quelle manière Rome »s'est érigée en capitale de la quatrième monarchie ${ }^{30}$. Certes, le mémoire de Lautz avait des objectifs pédagogiques, mais, malgré la brièveté qui lui était imposée, l'auteur formulait des idées nuancées sur la condition juridique des États territoriaux et sur l'Empire en général. Si Lautz a rédigé son mémoire pour l'éducation des enfants du duc de Noailles, ce n'est pas un cas unique. Dans la seconde moitié du XVII' siècle, Du May

traités d'histoire et de droit composés pour l'éducation des jeunes princes, cf., par exemple, Ass. nat. 234, »Positiones juris naturae et gentium in usum Serenissimi Principis Regii Poloniae et Saxoniae Francisci Xaverii conscriptae et ab eodem Serenissimo Principe annotationibus illustratae. Anno 1746 (titre, fol. 3).

${ }^{26}$ Cf. Deutsches Biographisches Archiv I 745, 137. Il ne faut pas le confondre avec Elias Christoph Lautz (Worms 1739-Wiesbaden 1798), qui était au service de la maison de Nassau, d'abord à Sarrebruck, puis à Wiesbaden, cf. ibid., III 548, 8.

27 AE, MD All. 66 fol. 8.

${ }^{28}$ Cf. ibid., fol. 91 : »ces états, quoyque ressemblans en tout aux parfaits souverains à cause de la conformité de leurs droits et privilèges, ne le sont pourtant qu'analogiquement étant sous la dépendance de l'Empereur et de l'Empire«.

29 Ibid., fol. 4.

30 Ibid. 
avait déjà publié un livre destiné aux jeunes princes pour leur apprendre le droit public allemand, ouvrage qui connut plusieurs éditions; Scheid fit de même au début des années 1750; Dupal composa un manuscrit à ce même effet, destiné aux princes royaux d'Angleterre. La connaissance du droit public allemand ne fut donc pas complètement négligée dans l'éducation des nobles entre 1648 et 1756. Le fait que le dépôt des Affaires étrangères conserve une copie de l'»Abrégé« de Lautz prouve d'ailleurs que ce genre d'écrits, souvent rédigés par de très bons connaisseurs du droit public allemand, trouvait aussi des lecteurs parmi les professionnels de la diplomatie.

Le baron Charles de Martens avait certainement raison de souligner le caractère personnel de la politique internationale dans la seconde moitié du XVIIe siècle et la première moitié du XVIII ${ }^{31}$. Cependant, il est incontestable qu'en 1648 les autorités politiques éprouvaient déjà le besoin de justifier publiquement leur politique étrangère ${ }^{32}$. Cette nécessité ne cessa de croître au cours de la période que nous étudions. Pour mieux comprendre les enjeux des écrits de propagande ${ }^{33}$, nous évoquerons deux brochures publiées en 1735, l'année de la paix préliminaire conclue par la France et l'empereur à Vienne. En effet, ces deux documents sont centrés sur la discussion des rapports qui existaient entre l'autorité de l'empereur et le pouvoir des états ou qui auraient dû exister entre eux dans un État idéal. Comme nous avons pu l'observer dès les premiers chapitres, ce sujet fut l'un des grands thèmes de la littérature française sur l'Empire à partir de l'ère de Richelieu et ne cessa de l'être jusqu'au milieu du XVIIII siècle.

Après l'analyse de ces deux tracts de 1735 , ce dernier chapitre aborde également un problème de l'histoire croisée: la critique des ouvrages allemands portant sur le droit public du Saint-Empire dans le »Journal des Savants« et celle des ouvrages français sur le droit constitutionnel de l'Empire dans la $»$ Litteratur des Teutschen Staatsrechts«, de Johann Stephan Pütter, ainsi que dans l'un des premiers journaux allemands de nouvelles littéraires du XVIII e siècle, les »Göttingische Gelehrte Anzeigen«, connu pour la qualité extraordinaire de ses comptes rendus, notamment d'ouvrages d'histoire et de jurisprudence.

Dans son édition des actes de la paix de Westphalie, Johann Gottfried von Meiern, l'un des meilleurs juristes allemands du XVIII e siècle, critiquait sévèrement les écrits que les Français publiaient fréquemment depuis l'époque de la paix de Münster. Selon lui, ces écrits, qui avertissaient les états de l'Empire du danger qu'ils encouraient de se faire asservir par l'empereur, empoisonnaient en effet les

31 Cf. Charles, baron DE MARTENS, Le guide diplomatique [...], 2 t. en 3 parties, Leipzig, Paris 51866 , t. I, préface, p. XII-XIII. Cependant, les négociateurs et les hommes d'État, voire les princes, devaient bel et bien, même à la fin du XVII et au début du XVIII eiècle, exposer, développer et faire valoir des faits et des arguments à l'appui de leur cause; à cet égard, Martens surestime certainement l'indépendance de la monarchie dite absolue.

32 Cf. REPGEN, Westfälische Friede und Öffentlichkeit.

${ }^{33}$ Pour la propagande sous l'Ancien Régime, cf. toujours l'étude classique de Joseph KLAITs, Printed Propaganda under Louis XIV. Absolute Monarchy and Public Opinion, Princeton (N. J.) 1976. 
relations entre le peuple allemand et la cour impériale. Même l'année où l'on signa les préliminaires de la paix de Vienne, en 1735, de tels écrits furent publiés en français.

\section{La part de la propagande: le »Testament politique « de 1735 ou le prétendu dysfonctionnement du système judiciaire du Saint-Empire}

Dans ses »Acta Pacis Westphalicae publica «34, Meiern évoque l'édition d'une petite brochure qui parut à Hambourg, en 1735. Cette brochure portait le titre de »Testament politique pour les interests du Corps germanique « 35 . Son auteur, dont l'identité précise nous échappe, affirme qu'il est sur le point de terminer une longue carrière - probablement d'homme d'État, de juriste ou de diplomate - et qu'il se propose de rassembler à ce moment de sa vie »les principes qu' un homme de [s]on métier \& de [s]a profession peut estimer necessaires au bonheur de la $\mathrm{Pa}$ trie « ${ }^{36}$. Pour lui, il s'agit de se justifier devant Dieu de son comportement dans une situation politique mettant en danger sa patrie, l'Allemagne, qui serait dans une position pouvant »operer sa ruine $\&$ la perte de sa Liberté ${ }^{37}$. Ce patriote se veut le champion d'un équilibre entre les pouvoirs étatique et impérial, alors que la balance pencherait plutôt, à l'époque où il écrit, en faveur de l'empereur. Selon lui, l'Empire est divisé en deux camps, les partisans et les adversaires du renforcement ou du maintien du pouvoir impérial; il considère cette partition, qui reflète dans le discours politique la bipolarité de la jurisprudence dans l'Empire, comme une anomalie ou, pour reprendre ses propres termes, comme la conséquence d'un »abus $\ll^{38}$.

Les partisans de l'empereur, tendant à transformer l'Empire en monarchie absolue, risquent d'écraser toute participation étatique; or, l'Empire connaît, selon cet auteur qui décrit ainsi parfaitement la réalité constitutionnelle, plusieurs niveaux de représentation ${ }^{39}$ : »Des partisans outrés de cette Autorité «, écrit-il en

${ }^{34}$ Cf. Merern, Acta Pacis Westphalicae publica, t. V, Vorbericht (non paginé), ici la première et la deuxième page, note $\left({ }^{*}\right)$. Selon Meiern, son auteur était un noble français qui avait été longtemps chargé de négociations très importantes en Allemagne.

35 Testament politique pour les interests du Corps germanique, Hambourg 1735; la brochure comprend onze pages de texte et un frontispice sans indication de l'éditeur ni de l'auteur. Au Quai d'Orsay, on peut trouver une édition publiée sine loco, avec une pagination différente, également de 1735 (AE, MD All. 74 fol. 311-318).

36 Testament politique, p. [3]. Ajoutons que l'auteur se dit lui-même un des »membres« de l'Empire, ibid., p. 4. En réalité, l'auteur était français (voir ci-dessus).

${ }^{37}$ Ibid., p. [3]. Le mot »Patrie « revient à plusieurs reprises dans cette brochure, et le »bonheur de la patrie« (ibid., et p. 4: »le bonheur de ma patrie«) est un leitmotiv dans les intentions de cet auteur.

38 Ibid., p. 4. Il se démarque ainsi du soi-disant »Testament politique du duc de Lorraine« de 1687, dont l'auteur a été identifié avec l'abbé de Chévremont.

${ }^{39}$ L'historiographie allemande parle aujourd'hui de plusieurs niveaux d'»État« (Einzelstaat et Bundesstaat), cf., par exemple, BURKHARDT, Das Reformationsjahrhundert. 
effet à propos des défenseurs des droits impériaux, »veulent qu'elle s'exerce d'une maniere si absoluë, qu'effectivement elle anéantiroit tous les degrez de representation qui lui sont inferieurs«. De la même manière, il se distingue de ceux qui défendent les seuls intérêts et droits des opposants à l'empereur et à sa maison; en effet, il estime que l'interprétation que ceux-ci font de la Constitution du Saint-Empire est aussi préjudiciable aux intérêts de l'Allemagne et des états qu'une vision purement monarchique, notamment parce que l'empereur assure la protection de ces derniers; protecteur des faibles contre les forts, il ne lui est pour autant pas permis d'empiéter sur les droits des ordres "auxquels, en ce point, elle [son autorité] doit être subordonnée $\ll^{40}$.

Pour lui, la distribution des pouvoirs dans l'Empire est toujours susceptible de modifications. À l'heure où il écrit, il estime que le pouvoir impérial a connu un essor très net, rendant nécessaire des restrictions supplémentaires, ou du moins la fortification de celles qui existent, pour assurer la liberté des états. D'après l'auteur du »Testament politique«, ce sont les lois fondamentales, notamment les traités de Westphalie et les capitulations impériales, qui représentent les moyens traditionnels propres à limiter le pouvoir impérial. En effet, les capitulations impériales gagnèrent en importance dans la pensée des auteurs du XVIII siècle, puisque les traités de Westphalie, qui passaient en France pour la loi fondamentale la plus importante du Saint-Empire depuis l'époque de Heiss et d'Obrecht, n'étaient pas assez précis sur certains points et avaient ignoré des réalités qui préoccupaient à présent les esprits. Pour cet auteur, ce sont donc ces capitulations qui, avec la paix de Westphalie dont elles constituent une sorte de mise à jour, assurent le respect des droits des états de l'Empire ${ }^{41}$.

Mais, à cette époque, ces lois fondamentales ne suffisent plus pour prévenir les transgressions visant à augmenter le pouvoir impérial au détriment des états. Au temps de la paix de Westphalie, continue l'auteur, »les Empereurs ne possedoient que ce que l'on nomme les Etats hereditaires d'Allemagne«. Ils ne pouvaient »entretenir qu'un nombre proportionné de troupes, $\&$ assez médiocres «. D'après lui, l'empereur n'avait donc à cette époque aucune emprise, aussi minime fût-elle, sur le reste de l'Empire. Admettons que la forme de la publication de cet auteur exigeait une description en noir et blanc plutôt qu'une analyse nuancée de la situation politique telle qu'elle se présentait respectivement au moment des traités de Westphalie et en 1735. Il n'en reste pas moins que cette description sous-estime le pouvoir de l'empereur en 1648, puisque celui-ci avait gardé ses attributs constitutionnels - la paix de Westphalie ne limitait en effet point ses droits de façon explicite $^{42}$.

On peut déduire de ces remarques que l'auteur considérait la situation en $\mathbf{1 7 3 5}$ comme inconstitutionnelle, et qu'à ses yeux l'empereur s'était arrogé un pouvoir qui ne lui revenait pas au regard des lois fondamentales du Saint-Empire. Et effec-

40 Testament politique, p. 4.

41 Ibid., p. 5.

42 Ibid. 
tivement, c'est avec une clarté qui ne prête point à équivoque qu'il s'attaque à la juridiction de la cour de l'empereur, c'est-à-dire aux jugements rendus par le Conseil aulique, dont la manière d'agir n'a, de son point de vue, plus rien à voir avec ce qu'elle était au milieu du XVIIe siècle, d'où résulte, toujours selon cet auteur, un très net dysfonctionnement de tout le système judiciaire et politique du SaintEmpire; à l'époque des traités de Westphalie, la »Jurisprudence de ce Tribunal n'étoit pas encore aussi monstrueusement differente de celle du reste de l'Empire « ${ }^{43}$. Cette $>$ monstruositér semble bien être une allusion à la définition que donne Pufendorf de la Constitution du Saint-Empire, qui était connue des publicistes allemands et français. Mais le sens de cette définition est ici détourné, puisque ce mot a sans aucun doute une connotation négative. Si la jurisprudence du Conseil aulique est "monstrueusement differente « de celle des autres tribunaux on doit en premier lieu penser à la Chambre impériale - l'unité et le bon fonctionnement du système judiciaire du Saint-Empire ne sont plus bien assurés. Au-delà de ce dysfonctionnement, tous les rapports de force se sont modifiés en faveur de l'empereur, tandis que l'union des princes et le soutien de la France et de la Suède assuraient jadis l'équilibre des pouvoirs étatique et impérial dans l'Empire.

L'auteur de la brochure se limite à étudier les causes des changements survenus, selon lui, depuis l'époque de la paix de Westphalie dans la Constitution du Saint-Empire; contrairement à d'autres publicistes, il ne propose pas de réforme du système politique allemand, estimant que "les remedes«, qui ne seraient par ailleurs pas faciles à trouver, »dépendent absolument des conjonctures « imprévisibles ${ }^{44}$. Il indique néanmoins la direction à suivre à l'avenir; car l'objectif de ce tract est bien entendu d'exercer une influence sur les décisions qui seront prises ultérieurement; il ne s'agit point d'une étude d'un genre académique qui veuille uniquement déterminer les causes de l'état actuel du Saint-Empire sans en déduire de conséquences concrètes.

À l'origine des changements que l'auteur a observés dans l'Empire, on retrouve, selon lui, la prétendue volonté de la maison d'Autriche de transformer l'Empire en véritable monarchie. Or, cet auteur estime que la monarchie n'est pas la forme de gouvernement fixée par les lois fondamentales. Toujours est-il que les moyens qui ont, d'après lui, permis à la maison d'Autriche d'étendre son emprise sur le reste de l'Empire ne constituent pas proprement des transgressions de ces lois fondamentales. L'auteur distingue trois catégories de moyens ou de causes qui ont abouti à augmenter la puissance de l'empereur dans l'Empire et au-delà de ses frontières: primo, la distribution d'honneurs et de prérogatives ainsi que les anoblissements et les gratifications, qui ont permis à l'empereur de diviser les états et lui ont notamment procuré une plus grande emprise sur les délibérations de la diète, en combinaison avec d'autres mesures destinées à renforcer son pouvoir à Ratisbonne, en particulier grâce à la dictature ${ }^{45}$; secundo, la tentative

43 Ibid., p. 5-6.

44 Ibid., p. 6.

${ }^{45} \mathrm{La}$ dictature était chargée de dicter les documents discutés ou rédigés à la diète. C'est 
d'éloigner les états de l'Empire de la France et de la Suède; tertio, la très nette amélioration de la position de l'empereur sur la scène européenne.

Il faut remarquer qu'une unité parfaite entre tous les ordres de l'Empire n'a jamais existé au moment de la conclusion des traités de Westphalie, ni probablement à aucune époque; l'auteur de cette brochure procède plus à la construction d'un état idéal projeté dans le passé qu'à la reconstruction d'une situation historique précise. Parmi les concrétisations de cette politique, cet auteur fait en particulier allusion à la création du neuvième électorat et aux autres dignités conférées par l'empereur, ainsi qu'à la réintégration pleine et entière de la Bohême au collège électoral en 1708.

Au lieu de reprocher à l'empereur certaines de ses mesures comme étant quasiment inconstitutionnelles, comme le fait cet auteur, on est obligé de souligner qu'au moins une partie d'entre elles étaient parfaitement conformes à la Constitution de l'Empire; en particulier, le droit d'anoblissement lui revenait incontestablement. On peut constater ici que des droits que Bodin avait méprisés, car il les considérait comme purement honorifiques et vidés de toute importance juridique, pouvaient en réalité constituer des moyens propres à asseoir la puissance impériale. De la même manière, la politique impériale menée à Ratisbonne ne paraît pas clairement inconstitutionnelle, contrairement à ce que suggère cet auteur, puisque l'empereur jouissait effectivement à la diète de droits que la Constitution de l'Empire lui accordait, comme par exemple le droit de proposition; ces droits furent également sous-estimés par Bodin. L'auteur de la brochure de 1735 n'examine pas la question de savoir quels droits devaient constitutionnellement revenir à l'empereur. Il insinue l'idée d'un procédé illégal en affirmant que le parti impérial »s'est rendu totalement maître de la Dictature à Ratisbonne. La Commission Impériale ne s'y borne plus à un ministère de protection \& de représentation, mais au contraire, elle n'y laisse proposer que les questions qui conviennent ou peuvent être agréables«. En réalité, il semble que ce sont plutôt les guerres de Louis XIV qui détruisirent les bonnes relations qu'entretenaient certains princes de l'Empire avec la France.

L'auteur dresse ensuite un tableau impressionnant de la puissance de l'empereur au niveau international, qui, en combinaison avec les progrès que la politique impériale a réussi à faire dans l'Empire même, contribue à rendre son autorité telle »qu'elle est plus que jamais, quand elle le voudra, en état d'empiéter sur les droits de nos Princes de l'Empire «46. Quelles sont les forces sur lesquelles se fonde une telle puissance de l'empereur, si formidable en Europe, en 1735 47 ? Cet auteur les résume ainsi: les empereurs sont, pour lui, les »Maîtres d'établissemens

nous qui avons opéré la répartition des différentes causes alléguées par cet auteur en trois catégories, afin de clarifier leur analyse.

46 Pour ces citations, voir Testament politique, p. 6-7.

47 L'intention de cet écrit est évidemment de faire surestimer la puissance impériale; néanmoins, il paraît intéressant de voir quelles raisons ont pu être alléguées, en 1735 , pour justifier ces assertions, puisqu'on doit supposer qu'elles devaient du moins être crédibles auprès des lecteurs visés par la brochure. 
très-considérables en Italie; Possesseurs de prèsque tout un côté de la Mer Adriatique; inattaquables du côté de la Hongrie, depuis que la Paix de Carlowitz a éloigné les Frontieres de l'Empire Ottoman; absolus prèsque dans le Nord, par l'effet que nous voyons de leurs Alliances avec la Russie; s'étendant, par les Païs-Bas, jusqu'à l'Océan «48.

Les trois catégories de raisons qu'il allègue correspondent presque parfaitement à un document diplomatique de la même époque ${ }^{49}$. Cela veut dire non seulement qu'un renforcement de la puissance impériale en Allemagne et en Europe, entre 1648 et le milieu des années 1730 , a été constaté par plusieurs auteurs de langue française, mais aussi qu'il y avait une liste de causes expliquant les changements intervenus, liste sinon canonique, du moins pas strictement personnelle. Parmi les causes plus proprement constitutionnelles, le droit de l'empereur d'accorder des privilèges a joué un rôle tout à fait fondamental pour ces auteurs; en outre, il faut remarquer que plusieurs auteurs constatent que l'empereur contrôle et domine les délibérations à la diète de l'Empire et que celle-ci, au lieu d'assurer la participation des ordres à la législation, est devenue un instrument de la politique impériale.

Selon l'auteur de la brochure de 1735, cette évolution interne et externe comporte le risque non seulement de transformer l'Empire en véritable monarchie (ou même en un despotisme), mais encore d'éliminer complètement le régime de la Landeshoheit en Allemagne, c'est-à-dire la spécificité de sa Constitution qui la distingue des monarchies voisines ${ }^{50}$. Selon cet auteur, l'avenir qui se dessine pour les états de l'Empire n'est au fond rien d'autre qu'une adaptation de l'Allemagne au système français de gouvernement et d'administration. Il va sans dire que ce sombre tableau doit servir l'intention de l'auteur - qui cherche à persuader les états de l'Empire que leurs véritables intérêts ne coüncident point avec ceux de l'empereur - et qu'il n'est donc pas à prendre au pied de la lettre si l'on veut en dégager la véritable conception que cet auteur se fait de l'Allemagne de son temps. En revanche, il n'est pas inutile de noter qu'une telle conception de l'avenir du Saint-Empire ne paraissait pas si invraisemblable que l'on n'eût pu espérer convaincre les lecteurs de sa pertinence.

S'il n'est pas évident de remonter de ces assertions aux véritables conceptions de l'auteur à propos de l'avenir constitutionnel du Saint-Empire, sa description de la Constitution idéale est explicitement formulée dans cette brochure. Évidemment, il n'estime pas que la tendance monarchique qu'il observe dans l'Empire et

48 Testament politique, p. 7.

$49 »$ Refflections sur les intérêts actuels des princes et états de l'Empire au commencement de février 1735 «, AE, MD All. 41 fol. 332-370'. Selon une note ajoutée à gauche du titre, il fut "<re >mis à monsieur <le> Garde des < Sce>aux le $5<$ fé > vrier 1735«, ibid., fol. 332. Dans l'analyse de ce document, l'on peut distinguer quatre catégories, du fait d'un ordre logique différant de la brochure, bien que les raisons qui y sont alléguées soient en principe les mêmes, de sorte que, malgré des différences mineures, on pourrait penser à une origine commune de ces explications.

${ }^{50} \mathrm{La}$ brochure ne parle pas des républiques. 
qui risque d'éliminer le pouvoir territorial des ordres soit le fondement sur lequel la Constitution allemande ait été établie. D'une manière positive, il caractérise ainsi cet état idéal, fondé notamment sur une protection mutuelle de l'empereur et des états et sur le respect des lois fondamentales:

Selon cette constitution, le Chef de l'Empire augmente la considération \& fait la sûreté du Corps qui l'environne, dont les membres doivent courir à la défense du Chef, quand il est injustement attaqué. Il est destiné à procurer justice à ceux de ses membres qui seroient injustement opprimez par d'autres. Mais la justice qu'il doit rendre ne doit point émaner d'aucune jurisprudence particuliere \& différente de celle de tout l'Empire.

Cette dernière remarque s'applique très certainement à sa critique des jugements récents rendus par le Conseil aulique. L'existence même d'un tel tribunal dépendant seulement de l'empereur semble constituer une anomalie pour cet auteur, car il poursuit en soutenant que l'Empire doit être gouverné "par ses propres loix «, et que »même dans les causes majeures il est avec l'Empereur partie nécessaire pour décider«. Cependant, en remettant en cause la compétence du Conseil aulique en de pareilles matières, cet auteur nie le bien-fondé d'une réalité constitutionnelle trop bien établie dans l'Empire pour être taxée d'abus de la politique impériale, à savoir la concurrence qui existait entre le Conseil aulique et la Chambre impériale.

En ce qui concerne le régime féodal, il distingue la personne de l'empereur de sa qualité de suzerain du Saint-Empire, arguant que ce «n'est point de la personne de l'Empereur, mais du chef représentant en ce moment toute la Société Germanique, que nos Princes prennent les investitures \& prêtent le serment « 51 . Pour cet auteur, les liens féodaux qui existent ne suppriment donc en aucune manière le droit de résistance, qui est même dans une certaine mesure un devoir de résistance, puisque c'est, le cas échéant, par leur opposition à l'empereur que les princes restent fidèles au serment qu'ils ont prêté.

Deux aspects sont essentiels, selon lui, pour assurer le bien public ${ }^{52}$ en Allemagne: le respect des restrictions que les lois (fondamentales) et la tradition imposent à l'autorité impériale, et l'union entre l'empereur et les ordres. Le non-respect de ces principes entraînerait de très graves conséquences. À part cela, il tient pour nécessaire une étroite union entre l'Empire et son chef; le terme d'»Empire« est donc pour lui synonyme des états de l'Empire ${ }^{53}$. Or, une telle union, »solide « et »utile à l'Empire «, ne peut être établie »qu'à l'abri des constitutions de l'Empire même «. La solidité de cette union et le respect des lois fondamentales dépendent donc mutuellement l'un de l'autre. Évidemment, l'intention de la brochure ne permet pas d'imaginer que les états de l'Empire pourraient eux aussi transgres-

51 Citations du Testament politique, p. 8.

52 L'auteur n'utilise pas ce terme mais parle de "bonheur « (ibid., p. 3 et 4) ou bien d'un Empire »grand \& heureux « (ibid., p. 8). De la même manière, l'on peut constater qu'il n'utilise pas le concept »équilibre «, que nous avons employé dans l'explication de son texte parce qu'il traduit bien la pensée de l'auteur.

53 Il dit explicitement: »L'Empire, à la vérité, ne peut être grand \& heureux qu'autant qu'il est étroitement uni avec son chef«, ibid. 
ser les limites de leur pouvoir constitutionnel; l'auteur n'invite donc les ordres à rechercher l'union avec l'empereur que dans la mesure où ce dernier se tient à ses constitutions, car, sans ce respect de la part de l'empereur, il n'en résulterait »qu'une fausse sécurité« et ce ne serait »qu'une tolérance dangereuse de ce qui pourroit mener la Cour Impériale à un despotisme absolu « ${ }^{54}$.

L'auteur n'hésite pas à reprendre des termes techniques latins du droit international et du droit public général, universellement compréhensibles dans l'Empire comme en France, quand il évoque les droits fondamentaux des états, consacrés par les traités de Westphalie, à la conservation et à l'exercice desquels il tient en premier lieu, précisant que si "les Traitez ${ }^{55}$ ont réservé à nos Princes ce qu'on nomme jura regalia, dont font partie jus legationis \& jus armorum, c'est qu'on avoit prévu que dans de certains cas nos Princes pourroient, pour leur propre conservation, avoir besoin d'en faire usage « ${ }^{56}$. L'auteur invite les princes de l'Empire à la vigilance face aux prétendues intentions de la cour de l'empereur, sinon d'abroger ces principes, du moins de les vider de leur sens en en empêchant l'exercice. Certes, il reconnaît que l'exercice de ces droits des ordres, qu'il qualifie de »suprêmes « ${ }^{57}$, tout en ne les confondant peut-être pas avec les droits de souveraineté58, ne doit pas être dirigé contre l'Empire ${ }^{59}$. Mais il n'évoque point la deuxième partie de cette restriction, qui interdit aux princes de contracter des alliances dirigées contre l'empereur, que ce soit avec d'autres princes de l'Empire ou avec des puissances étrangères. Il n'envisage même pas le cas ordinaire où l'empereur agit en considération de l'intérêt de l'Empire, car son objectif est de justifier le comportement des états qui vont contre l'empereur. Et cette tentative de justification regarde particulièrement les ordres qui refusent de participer à une guerre commune. Sans envisager la procédure ordinaire de la décision d'une guerre menée par l'Empire, qui inclut une décision de la diète, la brochure parle seulement de la volonté personnelle de l'empereur d'entreprendre une guerre. Dans ce cas-là, rien n'oblige les états, selon cette brochure, à entrer dans aucun engagement vis-à-vis de l'empereur: „Cette entiere liberté naît de ce qu'ils cessent de trouver un protecteur dans celui qui devroit l'être comme leur Chef « 60 .

Face à cette politique impériale, il recommande aux états de l'Empire de rechercher le soutien de la France (au moment où la succession de Pologne opposait ces deux puissances), "puisque c'est le seul appui solide, sur lequel nous puis-

54 Ibid.

55 Quoiqu'il ne précise pas de quels traités il parle en particulier, il ne peut s'agir que des traités de Westphalie, confirmés par les traités postérieurs.

56 Testament politique, p. 8-9.

57 Ibid., p. 9.

58 On peut supposer qu'il n'utilise pas l'expression »droits suprêmes« comme synonyme de »droits souverains« ou de »droits de souveraineté«, mais qu'il souligne par cette expression que ces droits sont tout à fait essentiels au fonctionnement de la Constitution de l'Empire. 59 Testament politique, p. 9. On peut préciser que cela est clairement interdit par l'article VIII § 2 de la paix d'Osnabrück et par le $\S 63$ du traité de Münster.

${ }^{60}$ Ibid. Cette source parle encore de l'»Empire« comme une entité opposée à l'empereur, cf. ibid. 
sions compter dans des cas, où nous aurions à réclamer les Traités de Westphalie \& les autres Constitutions de l'Empire«, opposant la politique de Louis XIV à la politique fondamentalement différente menée par la France depuis la paix d'Utrecht, de sorte que l'on ne devrait plus faire attention aux tentatives de la cour de Vienne de discréditer la France dans l'Empire ${ }^{61}$. Plus que l'empereur, le roi de France lui paraît donc le véritable protecteur de l'Empire. Sur ces fondements qu'il croit avoir établis, l'auteur critique ouvertement la récente décision prise par l'Empire de mener la guerre aux côtés de l'empereur. L'auteur estime que l'Empire n'a rien à gagner dans cette guerre, tout comme il n'avait tiré aucun profit de la guerre de la Succession d'Espagne, jetant seulement les bases, par sa participation, du »despotisme « de Léopold $\mathrm{I}^{\mathrm{e}}$, dont il craint, en 1735, »' augmentation « ${ }^{62}$. L'intention de la brochure est d'appeler les états de l'Empire à ne pas mettre en pratique la guerre décidée ensemble par l'empereur et les états.

Par l'analyse de cet écrit, on peut donc observer la persistance d'une conception dichotomique de l'Empire, qui limite la signification du concept "Empire" aux seuls états et oppose ces états à l'empereur, considéré comme un pouvoir quasi étranger à l'Empire et dirigeant ses forces contre les intérêts du pays. Mis à part cela, ce document passe sous silence certains éléments de la Constitution du Saint-Empire ou nie la validité de certaines stipulations, comme la défense d'une alliance contre l'empereur, ce choix se faisant à des fins de propagande. Le fait que des brochures de propagande ne retiennent que les éléments qui soutiennent le mieux leur argumentation, en laissant de côté ceux qui pourraient la contredire, n'est pas surprenant. En revanche, il n'est pas gratuit de noter que ce choix se fait sur une interprétation sélective des constitutions de l'Empire, et surtout il est intéressant de voir que certains aspects qui ne servent pas positivement cette argumentation ne peuvent pas être passés sous silence, comme la politique allemande de Louis XIV qui était encore trop bien ancrée dans les esprits pour qu'on puisse l'oublier, de sorte qu'une réfutation explicite de la validité de ses principes fut nécessaire en 1735.

${ }^{61}$ Après la mort du Roi-Soleil, on peut noter, dans les documents diplomatiques français, une autocritique de la diplomatie française au sujet de la politique mise en place par Louis XIV. En effet, en 1716, Le Dran examina les »Causes des préventions que le corps de l'Empire a prises contre la France sous le règne du Roy Louis XIV « de 1648 à 1700 . Ce mémoire mérite la plus grande attention étant donné l'objectivité avec laquelle il détermine les conséquences négatives de la politique de Louis XIV dans l'Empire et la clarté de son jugement, copie du mémoire, avec quelques corrections: AE, MD All. 52 fol. 110-114; le mémoire est daté du 20 juillet 1716 et explicitement attribué à $\gg N$. L. Le Dran, premier commis des Affaires étrangères « (fol. 110). L'aspect le plus important de ce mémoire, outre la capacité de voir les erreurs de la politique étrangère française, semble être le fait que l'auteur s'aperçoit du danger que la France représentait pour l'Empire. Cela contribua à surmonter les divergences internes et il apparut que le vrai clivage se situait entre l'Empire et la France et non pas entre les états de l'Empire et l'empereur.

62 Citations du Testament politique, p. 12 et 13. 


\section{La »Lettre à l'auteur du >Testament politique «« de 1735}

$\mathrm{Ce} \gg$ Testament politique « suscita une réponse: une petite brochure intitulée »Lettre à l'auteur du Testament politique «63. Cependant, ce n'est point une critique du »Testament«, mais bien un éloge de son auteur. Enfin, plus qu'une véritable réponse, on doit plutôt la tenir pour une continuation du »Testament politique«, qui interprète, sur la base des mêmes "principes « formulés dans ce "Testament«, la suite des événements qui se sont depuis déroulés dans l'Empire; ajoutons qu'elle semble bien avoir été tirée sur le même matériel typographique que ce »Testament «, de sorte qu'il n'est même pas exclu que les auteurs des deux brochures ne soient finalement qu'un seul personnage. Son intérêt est donc pour nous moindre que celui du »Testament«, parce qu'il s'agit surtout de questions d'actualité politique, comme dans un grand nombre de brochures, de tracts et de feuilles volantes qui, sans être inutiles à notre étude, sont des sources qui permettent mieux d'analyser l'instrumentalisation de l'imaginaire sur l'Empire que cet imaginaire même.

Les questions d'actualité politique et leur appréciation par cet auteur ne retenant pas notre intérêt, nous nous limiterons à dégager les éléments qui témoignent de sa conception de l'Empire. En ce qui concerne sa vision de l'Empire, il faut retenir qu'il part de l'idée d'un lien contractuel entre l'empereur et les états. Ce contrat doit être égal pour les deux parties contractantes, c'est-à-dire que, étant donné que »les Princes de l'Empire (terme par lequel l'auteur désigne généralement tous les états, comme cela se fait souvent en français) »ne peuvent faire aucun Traité qui tourne au préjudice de l'Empereur \& de l'Empire«, l'empereur ne peut pas réclamer le droit de conclure des traités pouvant nuire à l'Empire ${ }^{64}$. Or, l'auteur de la »Lettre« reproche ouvertement à l'empereur d'avoir rompu »publiquement la foi des Capitulations «, en permettant l'entrée de l'armée russe dans l'Allemagne ${ }^{65}$. C'est effectivement un problème pour lequel l'auteur peut se fonder sur des clauses précises dans les lois fondamentales de l'Empire pour soutenir son interprétation ${ }^{66}$; il se rapporte d'abord aux recès de l'Empire, qui »deffendent aux Empereurs de choisir des Etrangers pour commander des Armées en Allemagne«,

${ }^{63}$ Lettre à l'auteur du Testament politique, pour les interests du Corps germanique, Hambourg 1735; elle comprend six pages de texte et un frontispice. Sur ce deuxième écrit de propagande, cf. également MEIERN, Acta Pacis Westphalicae publica, t. V' Vorbericht (non paginé), ici la première et la deuxième page, note $\left({ }^{*}\right)$.

64 Lettre à l'auteur, p. 5. L'auteur de la »Lettre« parle, tout comme celui du »Testament«, de "Société Germanique « au lieu d'»Empire«, formule qu'il paraît avoir utilisée délibérément, dans ce contexte, puisque, comme aucune loi fondamentale de l'Empire ne contenait de clause correspondant à l'interdiction des alliances dirigées contre l'empereur ou l'Empire (article VIII $\$ 2$ du traité d'Osnabrück), il cherchait à imposer une obligation similaire à l'empereur en se fondant sur le droit naturel qui demandait l'équité des obligations dans "toute societé juste«, ibid. Dans cette perspective, le concept de »Société Germanique« semble avoir été employé pour contourner la discussion de la question qui consistait à savoir si ce principe devait être respecté dans l'Empire.

65 Ibid., p. 7.

66 En revanche, il est clair que cette interprétation constitue, non pas un examen désintéressé, mais une plaidoirie contre l'empereur. 
ensuite aux »termes formels des Capitulations Impériales«, dont il cite en français la promesse suivante de l'empereur: »Nous voulons ne faire entrer dans l'Empire aucune Troupe Etrangére sans la participation, conseil \& approbation des Etats de l'Empire, ou au moins des Electeurs « ${ }^{67}$. Quant aux dangers auxquels on s'expose en confiant aux armées étrangères la défense de son propre pays, cet auteur renvoie aux livres de Burgold ${ }^{68}$, de Bodin ${ }^{69}$ et de Burger $^{70}$. Selon cet auteur, l'objectif de l'empereur était d'épuiser les princes allemands par la guerre ${ }^{71}$.

Dans une plus forte mesure que le »Testament politique «, dont elle est probablement la continuation, cette »Lettre« condamne sans état d'âme la politique de l'empereur; le durcissement du ton s'explique par les événements de la guerre, qui laissent alors présager une issue peu favorable aux intentions de cet auteur ou des personnages qui sont éventuellement ses commanditaires. Les lois de l'Empire sont citées d'une manière sélective, ce qui permet d'accuser l'empereur de les avoir violées. Dans cette perspective, l'empereur, loin d'être le protecteur de l'Empire, est conçu comme son ennemi principal. Or, non seulement ces écrits, mais aussi les journaux littéraires visaient un public plus large.

\section{La critique des ouvrages allemands sur le droit public du Saint-Empire dans le »Journal des Savants«}

Le »Journal des Savants«, un périodique consacré aux lettres et aux sciences, publié à Paris et réimprimé à l'étranger, surtout à Amsterdam, était un journal d'une très grande renommée européenne ${ }^{72}$. Il rendait compte régulièrement des nouvelles publications dans le domaine de l'histoire, du droit et des sciences politiques. Outre les comptes rendus, ce journal publiait des listes de livres parus ré-

67 Lettre à l'auteur, p. 6.

${ }^{68}$ Ibid. Philipp Andreas Oldenburg (ou Burgoldensis, Burgold), juriste du XVII' siècle (mort en 1678), élève de Conring, cf. ZEDLER (éd.), Grosses vollständiges Universal-Lexikon, t. 4 (1733), col. 1975-1976, et t. 25 (1740), col. 1139.

69 Jean Bodin (1530-1596), fondateur de la doctrine moderne de la souveraineté.

70 Joachim Bürger (Burger), auteur des „Observationes Iuridico-Politico-militares«, publiées à Cologne en 1651, 1654 et 1675, cf. ZEDLER, Großes vollständiges Universal-Lexikon, t. 4 (1733), col. 1878.

${ }^{71}$ Lettre à l'auteur, p. 7-8.

72 Le Journal des Sçavans, périodique littéraire publié à partir de 1665, d'abord par le sieur de Hédouville [i.e. Denis de Sallo, conseiller au parlement de Paris]; nous avons consulté l'édition parue à Amsterdam à partir de 1676 (réimpression des tomes publiés depuis 1665). Pour la diffusion internationale des journaux français sous l'Ancien Régime, cf. La diffusion et la lecture des journaux de langue française sous l'Ancien Régime. Actes du colloque international, Nimègue 3-5 juin 1987, Amsterdam, Maarssen 1988 (Études de l'Institut de recherches des relations intellectuelles entre les pays de l'Europe occidentale au XVIIe siècle, Nimègue [SIB], 17), en particulier les articles d'Otto S. LANKHORST, Le rôle des librairesimprimeurs néerlandais dans l'édition des journaux littéraires de langue française (1684 1750), p. 1-9 et d'A. H. LAEVEN, La réception des plus anciens périodiques de langue française dans les pays allemands, p. 237-247. 
cemment. À travers ce formidable instrument de travail, les lecteurs français du »Journal des Savants « étaient donc au courant des nouveaux ouvrages juridiques publiés en Allemagne, même si seulement une partie de ces titres faisait l'objet d'une véritable recension. Dès le premier volume, le journal consacrait une partie de ses articles à l'histoire et au droit de l'Allemagne ${ }^{73}$. La généalogie des dynasties princières allemandes faisait également partie des intérêts du journal ${ }^{74}$, de même que la géographie de ce pays, qui intéressait les juristes et les historiens aussi bien que les militaires ${ }^{75}$. En outre, il rendait compte des nouvelles publications dans le domaine du droit des gens, de l'histoire universelle ainsi que des éditions des traités internationaux et des publications d'acta publica.

Pourtant, les véritables comptes rendus des ouvrages traitant du droit public allemand sont plutôt rares et souvent assez courts. Néanmoins, les rédacteurs ne manquaient pas de souligner certaines caractéristiques de la science du droit public en Allemagne. En 1666, on relève, par exemple, une allusion à la translatio Imperii: »Et parce que les Allemands veulent qu'on croye que leur Empire n'est qu'une suite \& une continuation de celuy des Romains, ils ne manquent jamais lors qu'il s'agit de leur Jurisprudence d'en prendre la source dans le droit Romain «76. Parmi les ouvrages analysés, nous retrouvons évidemment un grand nombre de titres qui ont été évoqués dans notre livre, tels que »L'Europe vivante « de Chappuzeau 77 et l'»Histoire d'Allemagne« par de Prade ${ }^{78}$. Cependant, beaucoup de livres n'apparaissent que dans les catalogues des nouveaux ouvrages, publiés à la fin de chaque année dans le dernier numéro du journal. Pour les premières années (1665-1677), l'édition d'Amsterdam regroupa tous les titres nouveaux dans une seule liste (à la fin du tome publié en 1678). Ce catalogue mentionne, entre autres écrits, l'»Estat de l'Empire«par Du May et celui de »Monzamban«, c'est-à-dire Pufendorf, ainsi que le $\gg$ Tableau historique « par Nouvelon ${ }^{79}$. Dans les comptes rendus et les catalogues des volumes suivants, nous retrouvons beaucoup d'ouvrages étudiés dans notre livre. On y relève, par exemple, les noms de Maimbourg ${ }^{80}$, de Rocoles ${ }^{81}$, de Varillas ${ }^{82}$. Nous y retrouvons également $» L e$ Pru-

${ }^{73}$ Cf. Journal des Sçavans, tome premier (1665-1666), p. 37-41.

74 Cf., par exemple, ibid., p. 405-406; tome second (1667-1671), p. 381-383.

75 Pour des publications concernant aussi bien la géographie générale du pays que ses fortifications, cf., par exemple, Journal des Sçavans, pour l'année M. DC. XCI. Tome dix-neufiéme, Amsterdam 1692, p. 189-190 et p. 604. Le compte rendu du volume sur l'Allemagne de »La Geographie anncienne, moderne \& historique « par d'Audriffet (1694) admettait la difficulté de ce sujet: »De tous les pays de l'Europe l'Allemagne est un de ceux qui fournissent la plus abondante matiere aux discours \& aux reflexions des Historiens \& des Geografes «, Journal des Sçavans, pour l'année M. DC. XCV. Tome vingt et troisiéme, Amsterdam 1696, p. 273-274 (citation p. 273).

76 Journal des Sçavans, t. I, p. 523-524, compte rendu de »Nomothesia Romano-Germanica seu de Constitutionibus Principum, Auctore Christ. Stirnio. Argentorati

77 Cf. ibid., t. II, p. 70-71.

78 Cf. ibid., tome cinquiéme (1677), p. 78-81 (article du 15 mars 1677).

79 Cf. ibid., „Catalogue Des Livres Nouveaux imprimez depuis l'an 1665 jusques à l'an 1677 inclusivement « (à la fin du volume).

80 Cf. ibid., tome septiéme (1679), p. 298-303. 
dent Voyageur « par Du May ${ }^{83}$, l'édition de la capitulation de Charles VII par Spon $^{84}$ et la »Capitulation harmonique « de Müldener dans la traduction fançaise de Besset de la Chapelle 85 .

Parmi les ouvrages sur le droit public allemand, on constate une préférence très nette du journal pour les titres latins. Alors que les Allemands étaient généralement connus pour leur goût des voyages et pour l'apprentissage des langues étrangères, en particulier du latin et du français, les Français, pour leur part, devaient souvent se débrouiller en parlant latin quand ils se trouvaient dans l'Empire ${ }^{86}$. D'ailleurs, l'obstacle que la lecture de livres allemands constituait pour le public français est explicitement évoqué dans le compte rendu que le »Journal des Savants« consacre à l'»Histoire d'Allemagne« par de Prade. Cette recension est d'une importance majeure, puisque l'auteur décrit le Saint-Empire comme un État monarchique bien que composé de différentes principautés et républiques (c'està-dire les villes):

Nous n'avons point d'Histoire plus embroüillée que celle d'Allemagne, tant par la diversité des évenemens qui s'y sont passez, \& le grand nombre de Principautez \& de Republiques, qui quelques differentes qu'elles soyent entre-elles, doivent pourtant former une méme Monarchie; que par le peu d'éclaircissement qu'en donnent les Historiens qui en ont écrit ${ }^{87}$.

Outre une description générale de l'ouvrage, le compte rendu fait un résumé des principales théories concernant l'origine du collège électoral. En effet, l'auteur souligne que ce problème était extrêmement contesté 88 .

81 Cf. ibid., p. 345.

$82 \mathrm{Cf}$. Journal des Sçavans, depuis le mois de juin jusques à la fin de l'année M. DC. LXXXVIII. Tome seizieme, Amsterdam 1689, catalogue des livres imprimés en 1688 (non paginé).

83 Cf. Journal des Sçavans, tome dixiéme (1683), p. 281.

${ }^{84} \mathrm{Cf}$. Journal des Sçavans, octobre 1743. Augmenté de divers articles qui ne se trouvent point dans l'édition de Paris. Tome CXXX, Amsterdam 1743, p. 279-280.

85 Cf. Le Journal des Sçavans, pour l'année M. DCC. LI, Paris 1751, p. 126 (février).

86 L'importance de la connaissance de l'allemand ou, à son défaut, du latin pour les militaires et les négociateurs français dans l'Empire est soulignée dans le compte rendu d'un dictionnaire trilingue (français, latin, allemand) paru à Francfort en 1688, cf. Journal des Sçavans, pour l'année M. DC. LXXXVIII. Suite du tome quinsieme, Amsterdam 1688, p. 342-343.

87 Et d'ajouter: "Le seul Lehmann qui est plus estimé de tous ayant donné son Histoire en une langue peu connüe en France «, Journal des Sçavans, t. X (1683), p. 78. L'auteur se réfère probablement à Christoph Lehmann, greffier de la ville de Spire au début du XVIIe siècle et auteur d'ouvrages historiques et de collections d'acta publica parfois réédités jusqu'au début du XVIII'; pour ce personnage, cf. ZEDLER, Großes vollständiges Universal-Lexikon, t. 16 (1737), col. 1428.

${ }^{88} \mathrm{Cf}$. Journal des Sçavans, t. V, p. [7]9: »De tous les points sur lesquels nous pourrions nous étendre, nous toucherons seulement celuy des Electeurs de l'Empire dont l'origine est encore tous les jours si fort contestée, les uns l'attribuant au Pape, les autres à la Puissance Imperiale, $\&$ les autres suivant une autre route que cet auteur croit la plus assurée «. Et d'exposer ces trois théories différentes. Le résumé des deux premières théories, réfutées par de Prade, reproduit, entre autres, les éléments fondamentaux de la fable des électeurs. Le résumé de la troisième théorie, à laquelle adhère de Prade lui-même, se fonde sur l'élection de Conrad III 
Le droit du Saint-Empire apparaît aux Français aussi compliqué (et parfois rebutant) que l'histoire de l'Allemagne, comme nous le voyons dans un compte rendu des »Institutiones juris publici romano-germanici« de Vitriarius: „Les Loix \& le droit Public d'Allemagne sont quelque chose de si mêlé à cause des differentes testes qui la gouvernent, $\&$ il naît de là tant de disputes $\&$ de difficultez pour les jeunes étudians qu'on jugera assez de l'utilité qu'ils pourront retirer de ces institutions « ${ }^{89}$. Parmi les auteurs des ouvrages analysés ou seulement mentionnés dans les listes, on trouve d'éminents juristes allemands, comme Carpzov et Leibniz.

Parfois, les comptes rendus donnent l'occasion d'une discussion sur certains problèmes fondamentaux du droit public allemand. Par exemple, à l'occasion d'une recension très précise consacrée à un traité latin sur la Landeshoheit, publié par un juriste allemand ${ }^{90}$, le "Journal des Savants « étudie avec une grande exactitude les doctrines concernant la superioritas territorialis des princes allemands, expression pour laquelle le journal utilise plusieurs traductions françaises différentes, par exemple »superiorité de territoire«, mais aussi »droit souverain de territoire « et »droit de territoire « tout court ${ }^{91}$. Mais ce livre suscitait aussi la critique de la part du »Journal des Savants«, parce que l'auteur prétendait que l'Empire était »une veritable Monarchie durant la vie de l'Empereur, \& une Aristocratie durant la vacance du Siege Imperial ${ }^{92}$. La publication de l'»Essai critique sur l'établissement et la translation de l'Empire d'Occident« par Guyon, en 1752, fournit l'occasion au »Journal des Savants « de discuter cette doctrine ${ }^{93}$.

Mis à part les comptes rendus des ouvrages déjà publiés, le »Journal des Savants« annonçait également les plans des livres dont la publication était prévue ${ }^{94}$.

à Coblence; il correspond presque parfaitement, voire littéralement, au récit que Heiss publia en 1684. L'élection impériale et les électeurs sont également évoqués dans le t. VI (1678), p. 189.

89 Journal des Sçavans, pour l'année M. DC. LXXXVI. Tome quatorsieme, Amsterdam 1687 , p. $523-524$.

$90 \gg$ De Jure Territorii« d'Andreas Knichen, publié à Francfort-sur-le-Main en 1688 avec deux autres traités concernant des problèmes similaires. L'auteur, Andreas Knichen (15601621), qui était chancelier de Thuringe, voulait renforcer le pouvoir territorial. C'est avec son grand ouvrage sur le droit territorial, publié d'abord en 1600, que, dans l'Empire, commence la formation de la doctrine de la superioritas territorialis. Cf. STOLleIS, Histoire du droit public, p. 216-217 et p. 275-276.

91 Cf. Journal des Sçavans, t. XV, 1688, p. 360-371, deuxième citation p. 361, première citation p. 369, troisième passim (aussi au pluriel). À noter que dans un autre compte rendu publié dans le même volume, p. 586-590, l'auteur dit que le livre analysé présente des notices sur les princes d'Allemagne qui régnaient alors et sur les autres seigneurs »joüissant de droits Royaux « (citation p. 586). La terminologie du »Journal des Savants « était donc aussi variée à cet égard que les traductions françaises des lois fondamentales du Saint-Empire et des traités internationaux.

92 Ibid., p. 361.

93 Cf. Journal des Sçavans, édition de Paris 1753, p. 395-401 (article du 15 juin) et p. 841847 (article du 15 décembre); la publication de cet ouvrage avait été annoncée ibid., p. 61 (en janvier).

94 Sur le plan d'une Histoire d'Allemagne en trois volumes, cf. Joumal des Sçavans, août 
Il fournissait aussi l'occasion aux libraires d'attirer l'attention du public cultivé sur la possibilité de lui procurer des livres étrangers, par exemple en provenance de Francfort ${ }^{95}$. En Hollande, les différents journaux français de critique littéraire étaient parfois publiés ensemble. Le »Journal des Savants « était par exemple relié avec les »Mémoires de Trévoux «. À partir de 1764, on y joignit même des extraits $\mathrm{d}^{\text {'autres journaux français et anglais }}{ }^{96}$.

Dans la critique littéraire française du milieu du XVIII ${ }^{e}$ siècle, la parution de la première édition de l'»Abrégé chronologique " de Pfeffel faisait l'objet d'un compte rendu très détaillép7. La traduction française du »Tableau du gouvernement actuel de l'Empire d'Allemagne « de Schmauss était également analysée, de même que les »Annales de l'Empire « de Voltaire, quoique très brièvement ${ }^{98}$. Ces trois comptes rendus étaient tirés des »Mémoires de Trévoux«.

En ce qui concerne l'ouvrage de Schmauss, le compte rendu approuve entièrement les positions prises par le professeur de Göttingen. On estime que son livre traite tous les aspects importants du sujet, que l'auteur a raison de joindre des considérations historiques à la jurisprudence et on apprécie en particulier qu'il évite de prendre »le ton de Politique«, en se limitant à instruire son lecteur. Le travail du traducteur est également loué: on avoue qu'il »mérite aussi des éloges, surtout pour le soin qu'il a pris d'éclaircir le Texte dans les Notes placées aux marges «99.

1743. Augmenté de divers articles qui ne se trouvent point dans l'édition de Paris. Tome CXXX, Amsterdam 1743, p. 558. Cf. également, pour les traités internationaux, le compte rendu du catalogue de l'édition des traités de paix publié par Léonard en 1690 dans Journal des Sçavans, pour l'année M. DC. XC. Tome dix-huitiéme, Amsterdam 1691, p. 699. Le journal annonçait que Léonard cherchait un auteur pour rédiger une histoire des traités de paix, qui devait être publiée dans le cinquième volume de sa collection de traités. Par ailleurs, le troisième volume des traités de paix passés avec les états de l'Empire, que Léonard publia en 1693, fut analysé dans Journal des Sçavans, pour l'année M. DC. XCIII. Tome vingt et uniéme, ibid. 1694, p. 322-323. Un »Abrégé chronologique « de l'histoire de l'Allemagne (certainement le livre de Pfeffel) était annoncé dans Journal des Sçavans, édition de Paris (1753), p. 252.

95 Cf. l'annonce du libraire Guillyn publiée ibid., p. 637.

96 En ce qui concerne la critique littéraire dans la deuxième moitié du XVIIIe siècle, il est intéressant de noter que dans les »Nouvelles Littéraires«, Strasbourg (comme lieu de publication d'ouvrages latins) est classée parmi les villes d'Allemagne, ce qui témoigne de la perception du rôle de la ville comme point d'échanges culturels entre l'espace germanique et le royaume de France, cf. Journal des Sçavans, avec des extraits des meilleurs journaux de France \& d'Angleterre. Suite des CLXX. volumes du Joumal des Sçavans, \& des LXXIX. volumes du même Journal combiné avec les Mémoires de Trévoux. Janvier 1764. Tome 1. No. I, Amsterdam 1764, p. 119-122.

${ }^{97}$ Cf. Journal des Sçavans, combiné avec les Mémoires de Trévoux. Suite des CLXX volumes du Journal des Sçavans, Amsterdam 1755, t. X (mars 1755), p. 149-156.

98 Cf. ibid., t. XI, vol. I (mai 1755), p. 123, et t. XII (juillet 1755), p. 527-528 (pour Schmauss); ibid. t. XI, vol. I (mai 1755), p. 124-127 (pour Voltaire). Étant donné que la rédaction n'avait pas ce dernier livre, on se limitait à signaler son intérêt.

99 Cf. ibid., t. XII (juillet 1755), p. 527 (citations) et p. 528 . Toutefois, ces notes paraissent parfois trop longues et peu claires, et l'introduction du traducteur n'est pas toujours approuvée, par exemple quand il définit le despotisme conformément à l'»Esprit des lois«, ibid., p. 528 . 
L'ouvrage de Pfeffel est considéré comme concis et très précis. Le compte rendu énumère les avantages de cet ouvrage, mais ne cache pas certaines faiblesses, comme l'inexactitude d'un certain nombre de citations ${ }^{100}$. Le $"$ Journal des Savants« rendait aussi compte de la traduction allemande de l'»Histoire d'Allemagne « que le père Barre avait d'abord publiée en français ${ }^{101}$; on le considérait comme l'ouvrage »le plus complet« sur son sujet ${ }^{102}$. En annonçant la dernière livraison, le journal fit un véritable éloge de cette traduction à cause de son exactitude ${ }^{103}$.

Outre le droit et l'histoire de l'Allemagne, les critiques littéraires faisaient aussi une place de choix aux différents États territoriaux allemands. Au milieu du XVIII ${ }^{e}$ siècle, la Prusse était évidemment placée au premier rang104. À la fin du XVIIe siècle, le »Journal des Savants « s'était intéressé, notamment, à la géographie du cercle de Souabe ${ }^{105}$, qui était alors une zone d'affrontements militaires entre l'Empire et la France. Les cercles et les territoires de l'Empire n'étaient donc pas négligés. Dans le »Journal des Savants«, les lecteurs français étaient renseignés sinon d'une manière exhaustive, du moins d'une façon à peu près régulière sur les principales publications concernant le droit public du Saint-Empire. Voyons maintenant comment les auteurs français qui écrivaient sur cette matière étaient jugés en Allemagne.

\section{La critique des ouvrages français sur le droit constitutionnel de l'Empire dans la »Litteratur des Teutschen Staatsrechts«, de Johann Stephan Pütter}

Entre 1776 et 1783, Johann Stephan Pütter, qui était conseiller privé à Göttingen, publia une bibliographie du droit constitutionnel allemand en trois volumes, très appréciée par les publicistes du Saint-Empire et qui l'est encore par les juristes et les historiens de nos jours ${ }^{106}$; l'auteur y répertoria non seulement les ouvrages qui

100 Cf. Journal des Sçavans, t. X (mars 1755), p. 149-156, en particulier p. 154-155.

$101 \mathrm{Cf}$. BARRE, Histoire générale d'Allemagne; ID., Allgemeine Geschichte von Deutschland.

102 Cf. Journal des Sçavans, édition de Paris (1751), p. 632, 668-669; ibid. (1752), p. 165171 (citation p. 166), 443; ibid. (1754), p. 380.

103 Cf. ibid.: »Cette traduction dédiée à l'Archiduc Joseph, paroît exacte surtout dans les derniers volumes: la plûpart des notes dont elle est ornée, sont plus sçavantes que critiques; quelques-unes regardent des points de droit public, omis par le P. Barre, dont la discussion lui avoit paru devoir être réservée aux publicistes Allemans «.

104 Cf., par exemple, Journal des Sçavans, combiné avec les Mémoires de Trévoux, t. X (avril 1755), p. 392 (droit prussien) et p. 394 (histoire du Brandebourg); ibid., t. XI, vol. II (mai 1755), p. 535 (histoire du Brandebourg).

105 Cf. Journal des Sçavans, pour l'année M. DC. XCIV. Tome vingt et deuxiéme, Amsterdam 1695 , p. 379-380.

106 Johann Stephan Pïtter (1725-1807), professeur de droit à Göttingen depuis 1746, suc- 
avaient été écrit en allemand sur ce sujet, mais aussi les ouvrages en langue étrangère et en particulier en français ${ }^{107}$.

Une remarque que Pütter fait sur Scheid montre qu'en général il n'estime pas beaucoup les auteurs français, puisqu'il dit que Scheid fait preuve de connaissances plus précises, en ce qui concerne les affaires d'Allemagne, que ne le font les Français en règle générale; mais, ajoute-t-il, on voit bien que Scheid n'a pas écrit cet ouvrage ${ }^{108}$ dans sa langue maternelle ${ }^{109}$. Or, les auteurs français reprochaient eux-mêmes à leurs prédécesseurs de s'intéresser peu aux pays étrangers et aux langues étrangères; mais leur objectif était de justifier leurs propres publications sur l'Allemagne, et d'y attirer l'intérêt du public. Pütter évoque une remarque de ce genre que Le Coq de Villeray fait sur ses compatriotes, dans la préface de son »Traité historique «110; Pütter met en relief que Le Coq, qui selon ses propres assertions se fondait sur les meilleures sources, communiqua son manuscrit à Schœpflin avant de le faire imprimer, et qu'il tint compte de sa critique ${ }^{111}$. Mais étant donné que Le Coq visait seulement un public français, pour lui donner quelques notions fondamentales de la Constitution allemande, sans prétendre écrire un ouvrage digne d'être lu en Allemagne, Pütter se montre indulgent à son égard 112 . Toutefois, il se limite à admettre que Le Coq donne "de temps en temps des descriptions pertinentes «113. Par contre, Pütter lui fait un reproche qui touche à un point fondamental: selon son opinion, cet auteur ne fait pas toujours bien la différence entre l'Empire médiéval et l'Allemagne moderne; de plus, Pütter estime que Le Coq va souvent trop loin en remontant jusqu'à Tacite pour retrouver les origines de certains faits ${ }^{114}$. La conclusion de Pütter n'est pas flatteuse; après avoir cité les recommandations simplistes que Le Coq propose pour ramener les protestants allemands à la foi catholique, il estime que les erreurs de cet auteur sont suffisantes pour que l'on ne soit plus curieux de rechercher ce livre, qui était très rare en Allemagne ${ }^{115}$. Pütter a raison de porter ce jugement sur le traité de Le

cesseur de Johann Jacob Schmauss en 1756 (chaire de l'histoire du Saint-Empire), était le juriste le plus célèbre de son temps dans l'Empire. Pour la vie et l'œuvre de Pütter, cf., entre autres, STOLLEIS, Histoire du droit public, passim (voir l'index, p. 633); Martin OTro, article „Pütter, Johann Stephan«, dans: Neue deutsche Biographie, t. 21 (2002), p. 1-2 (avec une bibliographie des œuvres et des études).

107 PUTTER, Litteratur des Teutschen Staatsrechts.

108 SCHEID, Traité sistématique.

109 PÜTTER, Litteratur des Teutschen Staatsrechts, t. II, p. 81: »Seine Kenntnisse von deutschen Sachen sind richtiger, als man sie bey Franzôsischen Schriftstellern erwarten kann. Es lẳ sich aber auch nicht verkennen, daß es nicht die Muttersprache war, worinn er schrieb«. ${ }^{110}$ LE CoQ, Traité.

111 PÜTTER, Litteratur des Teutschen Staatsrechts, t. II, p. 79.

112 Ibid.: $\gg$ Doch bescheidet er sich, daß er nicht fúr Teutschland, aber gut gnug für Frankreich geschrieben habe, um seinen Landsleuten nur einige nåhere Begriffe von der Teutschen Verfassung beyzubringen«.

113 Ibid.: "Hin und wieder gibt er ganz treffende Beschreibungen«.

114 Ibid. Pütter allègue à titre d'exemple les remarques de Le Coq sur les austrègues, ibid., n. a (cf. LE COQ, Traité, p. 287).

115 Cf. PÜTTER, Litteratur des Teutschen Staatsrechts, t. II, p. 79-80, n. a. 
Coq, qui n'avait pas sa place en Allemagne, où on disposait de meilleurs livres pour s'informer de la Constitution impériale; mais Pütter avoue lui-même que ce n'est pas le public allemand qui était visé par cet auteur. En revanche, Le Coq pouvait effectivement expliquer des notions fondamentales du droit public d'Allemagne au public français cultivé, sans être spécialiste des problèmes juridiques, et cela correspondait tout à fait à son intention; car malgré certaines erreurs, il n'est pas vrai que cet ouvrage en soit particulièrement parsemé.

L'auteur français suivant qui est critiqué par Pütter est Éléazar de Mauvillon, qui publia son $\gg$ Droit public germanique« en $1749^{116}$, un an après la publication du traité de Le Coq. Comme cet ouvrage avait été imprimé sans nom d'auteur à Amsterdam, Pütter tira le nom de Mauvillon d'une annonce de son livre; selon les informations dont il disposait, Mauvillon était professeur de français au Carolinum de Brunswick ${ }^{117}$. À propos d'une remarque de Mauvillon, qui dit, dans la préface de son livre, qu'il est "pour le moins autant Allemand que François«, Pütter note que cet auteur renvoie effectivement assez souvent, en marge de son texte, à des juristes allemands, comme Bilderbeck, Moser et Schmauss ${ }^{118}$, et qu'il semble donc bien connaître ces auteurs. Pütter conclut qu'il est incontestable que Mauvillon connaissait l'Allemagne mieux que Le Coq, tout en reprochant aux deux auteurs français que de nombreuses erreurs se soient glissées dans leurs ouvrages $^{119}$.

Pfeffel est l'auteur français suivant sur lequel Pütter se penche; on peut s'étonner de la brièveté des remarques de ce dernier sur cet auteur, étant donné son importance, mais Pütter a raison de souligner que Pfeffel a écrit un ouvrage ${ }^{120}$ qui est plutôt une histoire de l'Allemagne qu'un traité sur son droit public, et que cette histoire est calquée sur le modèle de l'histoire de France par Hénault, même si l'auteur y a inséré des notes sur le droit public des siècles passés ${ }^{121}$. Ce jugement est sans appel. Toutefois, l'évolution du droit public constitue le sujet principal du récit historique de Pfeffel. Notons, par ailleurs, que Pütter tient cet ouvrage pour réussi (littéralement, il dit "glücklich« [heureux]), et qu'il n'y relève aucune faute.

116 MaUVILlON, Le Droit public germanique (1749; nouvelle édition en 1756).

117 PÜTTER, Litteratur des Teutschen Staatsrechts, t. II, p. 80.

118 Ibid. Christoph Lorenz Bilderbeck était notamment l'auteur d'une bibliographie du droit public germanique (Bibliotheca iuris publici Germanici, publiée en 1715 et en 1738), cf. ibid., t. I, p. 23.

119 Ibid., t. II, p. 80.

120 Pfeffel, Abrégé chronologique. Pütter en connaît seulement quatre éditions sur cinq; l'édition de Paris de 1766 lui a échappé. Cf. PÜTTER, Litteratur des Teutschen Staatsrechts, t. II, p. 83.

${ }^{121}$ Ibid. Charles-Jean-François Hénault (1685-1770), président de la première chambre aux enquêtes du parlement de Paris en 1710, membre de l'Académie française en 1723 et de l'académie de Berlin en 1749, était l'auteur de l'»Abrégé chronologique de l'histoire de France «, plusieurs fois édité aux XVIII et XIX e siècles, cf. CioRanESCU, Bibliographie de la littérature française du dix-huitième siècle, t. II, p. 937-939. 
En 1755, ce fut le comte Louis Gabriel du Buat qui traduisit Schmauss en français, et cette version française n'a pas échappé à la critique de Pütter ${ }^{122}$. Cependant, Pütter ne dit rien sur la qualité de la traduction, se limitant à signaler que le traducteur y ajouta certaines notes concernant notamment le Moyen Âge. Pütter signale également le traité publié par le même auteur (Du Buat-Nançay) en 1757, en quatre tomes, sur $»$ Les origines ou l'ancien gouvernement de la France, de l'Allemagne et de l'Italie «. Toutefois, il se limite à une remarque sur la biographie de cet auteur, signalant qu'il avait été envoyé français à Ratisbonne, puis à Dresde, avant de retourner en France en 1774. Mais Pütter ne rend pas compte des forces et des faiblesses de cet ouvrage ${ }^{123}$.

Ce livre fut publié après 1756. Ajoutons cependant une remarque sur deux publications françaises postérieures qui furent également critiquées par Pütter: il s'agit des livres de Gérard et de Schmettau. En ce qui concerne Gérard, un Alsacien qui avait été secrétaire de l'ambassade française à Dresde ${ }^{124}$ et qui était l'auteur d'un traité sur le droit public ${ }^{125}$ qui suivait le plan de l'ouvrage de Mascovius, le compte rendu de Pütter reprend la critique faite par Moser; ce dernier avait souligné qu'il s'agissait d'un livre écrit avec modestie et qui devait passer pour le meilleur ouvrage sur ce sujet en langue française, tout en lui reprochant des imperfections essentielles ${ }^{126}$. L'»Abrégé du droit public d'Allemagne « 127 contenait un "Discours préliminaire « très long, qui était en réalité une véritable histoire de l'Allemagne. Pütter reproduit les titres des douzes chapitres qui composaient ce livre. Étant donné qu'il n' avait pas vu cet ouvrage publié en 1778, il résumait seulement ce qu'il avait lu dans un autre compte rendu: celui-ci louait ce livre, puisqu'il était utile et écrit avec sincérité, tout en lui reprochant des erreurs grossières 128 .

Certes, tous les auteurs des ouvrages français dont Pütter rendait compte n'étaient pas français. D'ailleurs, certains auteurs ne publiaient pas uniquement en français. Par exemple, Charles-Frédéric Necker, qui était professeur à Genève, rédigea un livre sur le droit public en allemand lorsque le prince héréditaire de Hesse-Cassel se trouvait en Suisse. Quand Pütter publia sa bibliographie du droit public, ce prince était landgrave de Hesse ${ }^{129}$. D'après le jugement de Pütter, cette

122 SCHMAuss, Tableau du gouvernement actuel de l'Empire (1755). Cf. PÜTTER, Litteratur des Teutschen Staatsrechts, t. II, p. 84.

123 [Louis-Gabriel, comte Du BUAT-NANÇAY], Les origines ou l'ancien gouvernement de la France, de l'Allemagne et de l'Italie [...], 4 vol., La Haye, Paris 1757. Cf. PüTTER, Litteratur des Teutschen Staatsrechts, t. II, p. 84; cf. aussi ibid., t. I, p. 32.

124 À l'époque de Napoléon Ier, comme on le sait bien, ce personnage joua un rôle très important dans la politique allemande de la France.

125 [Joseph-Mathias GÉRARD DE RAYNEVAL], Institutions au droit public d'Allemagne, Leipsic, Zullichau 1766; réimprimé à Strasbourg, en 1771.

126 PUTTER, Litteratur des Teutschen Staatsrechts, t. II, p. 87; et de signaler les comptes rendus publiés, entre autres, par Moser.

127 [SCHMETTAU], Abrégé du droit public d'Allemagne.

128 Cf. PÜTTER, Litteratur des Teutschen Staatsrechts, t. II, p. 97-98, en particulier n. *.

129 Le tome I parut en 1776. Frédéric II (1720-1785) était devenu landgrave de Hesse-Cassel en 1760 . 
méthode ${ }^{130}$ était un ouvrage exquis, qui se fondait essentiellement sur la critique d'un autre juriste, Cramer ${ }^{131}$. Selon Pütter, Necker traitait des matières de droit public les plus courantes dans un allemand très pur; toujours selon Pütter, le traité de Necker combinait parfaitement la brièveté avec la clarté, distinguait clairement le droit moderne de l'Empire de son histoire et ne rapportait pas les idées erronées des auteurs précédents, révélant d'emblée la vérité à son lecteur ${ }^{132}$. En ce qui concerne le principal ouvrage français écrit par le même auteur (la »Description du gouvernement present du Corps germanique « $)^{133}$, Pütter se limite à signaler qu'en attribuant à Necker ce livre anonyme traduit en allemand en $1764^{134}$ il se fonde sur un compte rendu publié par Moser ${ }^{135}$.

Or, mis à part les ouvrages traitant du droit public allemand en français, les Français disposaient-ils aussi de bibliographies afin de repérer les publications sur ce sujet non seulement en français, mais aussi en latin? Notons que, selon l'opinion de Pütter, la »Methode« de l'abbé Lenglet Dufresnoy ${ }^{136}$ constituait un bon moyen à cet effet: ses notes sur l'étude du droit public, l'état de l'Empire et la liberté germanique contiennent des catalogues presque exhaustifs des ouvrages concernant l'histoire et le droit d'État de l'Empire, et l'auteur y ajoute aussi son propre jugement; pour cette raison, même Moser se rapporte à plusieurs reprises à Lenglet dans sa bibliographie du droit public ${ }^{137}$.

On voit bien que les jugements que Pütter porte sur les auteurs français, abstraction faite de Necker et de Pfeffel, sont plutôt sévères; doit-on en conclure à la mauvaise qualité des ouvrages qu'ils ont rédigés? Certes, des auteurs comme Le Coq, qui écrivaient pour un public cultivé, étaient trop superficiels pour plaire à Pütter. Par ailleurs, ce dernier ne mâche pas non plus ses mots quand il s'agit de critiquer les auteurs allemands. Or, la plupart des comptes rendus que nous venons d'analyser font partie du catalogue des livres qui ont été publiés sur le droit public allemand depuis 1746, et qui n'ont pas été publiés à Göttingen ${ }^{138}$. Sur les vingt-et-un paragraphes qui composent ce catalogue, sept concernent des publications en langue française, un paragraphe regarde la version allemande d'un traité

130 Elle fut publiée à Marbourg, en 1741, sous le titre de: Kurze und aus den eigentlichen rechten Quellen hergeleiteter Unterricht in dem Staatsrecht des H. R. R. Teutscher Nation, mit einer Vorrede; d'après Pütter, il s'agit d'un petit volume in- $8^{\circ}$. Cf. PÜTTER, Litteratur des Teutschen Staatsrechts, t. I, p. 476-477.

131 Johann Ulrich Cramer (1706-1754), notamment connu pour avoir transmis la philosophie de Christian Wolff dans la jurisprudence; sur sa vie, voir ibid., p. 443-445.

132 Cf. ibid., p. 477.

133 La première édition date de 1741 .

134 [Carl Friedrich NECKER], Beschreibung der gegenwårtigen Regierung des Teutschen Staats, insgemein das heil. Röm. Reich genannt, nach dem Französischen, Francfort, Leipzig 1764 , in- $8^{\circ}$.

135 PÜTTER, Litteratur des Teutschen Staatsrechts, t. I, p. 477.

136 [LENGLET-DufRESNOY], Methode pour etudier l'histoire (1713); sur les autres éditions, voir CioRANESCU, Bibliographie de la littérature française du dix-huitième siècle, t. II, 10921094; Pütter se réfère à la nouvelle édition de la »Methode«, parue à Paris, en 1735, 3 t. in-4º . 137 Cf. PÜTTER, Litteratur des Teutschen Staatsrechts, t. I, p. 24.

138 Cf. ibid., t. II, p. 78-98. 
français sur le gouvernement de l'Allemagne ${ }^{139}$, et treize paragraphes parlent des publications rédigées par des auteurs allemands en allemand ou en latin; à part la France, aucune nation étrangère n'est représentée dans ce catalogue.

Cela veut dire que, même si l'accueil que Pütter réserve aux ouvrages en langue française est plutôt réticent, ce catalogue rend un témoignage incontestable du rôle primordial que la France jouait dans ce domaine, car aucune nation (abstraction faite de l'Allemagne) ne pouvait rivaliser avec sa production de travaux sur le droit public germanique à la veille de la guerre de Sept Ans et au-delà de cet événement.

\section{La critique des ouvrages français sur le droit public allemand dans les »Göttingische Gelehrte Anzeigen«}

Les »Göttingische Gelehrte Anzeigen« rendaient également compte de certains ouvrages français portant sur le droit public du Saint-Empire ${ }^{140}$. En particulier, on peut y trouver des recensions d'une très grande qualité de l'ouvrage d'Éléazar de Mauvillon, en 1749, et des différents tomes du "Traité sistématique« de Scheid, entre 1751 et 1754. Le dernier compte rendu ne critique pas seulement l'œuvre de Scheid, mais offre également un aperçu critique général de la littérature française sur le Saint-Empire. C'est à cause de cette rétrospective, qui donne à cette recension un caractère plus général, que nous l'étudierons avant le compte rendu de Mauvillon.

La recension du »Traité sistématique« de Scheid comprend quatre parties. Chacune porte sur l'un des quatre volumes de l'ouvrage du juriste allemand qui écrivait en français ${ }^{141}$. La première partie de ce compte rendu rappelle d'abord les éléments autobiographiques contenus dans la préface du premier volume du »Traité sistématique«. En effet, Scheid avait préféré gardé l'anonymat aussi bien sur le frontispice de son livre que dans sa dédicace au mécène de l'académie de Göttingen. La préface constituait donc la seule source permettant de savoir quelque chose sur l'auteur qui voulait faire connaitre le droit public allemand à des personnes qui ne maîtrisaient pas forcément l'allemand et le latin. Selon le jugement des »Göttingische Gelehrte Anzeigen«, l'auteur du »Traité sistématique» pouvait être sûr non seulement d'atteindre son objectif principal, qui était de plaire aux lecteurs étrangers, mais aussi de trouver des lecteurs dans l'Empire,

139 Il s'agit de la version allemande de l'ouvrage de Necker publié en français en 1740.

140 Ce périodique changea de titre au cours de la période qui nous intéresse; jusqu'en 1752 , le titre exact fut »Góttingische Zeitungen von Gelehrten Sachen«; à partir de 1753, le joumal s'intitula »Gơttingische Anzeigen von gelehrten Sachen unter der Aufsicht der Kónigl. Gesellschaft der Wissenschaften «.

141 Góttingische Zeitungen, année 1751, p. 387-395; ibid., année 1752, p. 302-308; Gôttingische Anzeigen, année 1753, vol. I, p. 131-135; année 1754, p. 345-346. Dans la dernière partie du compte rendu (1754), le critique estime que la traduction française de la paix d'Osnabrück de Scheid est la meilleure version française jamais publiée de ce traité, ibid., p. 346. 
puisque la langue française jouissait d'un tel éclat chez les Allemands que les plus prestigieuses cours princières s'en servaient même dans les affaires de l'État ${ }^{142}$. Il est vrai que la culture et la langue françaises étaient à leur apogée en Allemagne à la veille de la guerre de Sept Ans.

Le journal de Göttingen concède que le livre de Scheid n'était pas le premier ouvrage français sur le droit public allemand. L'auteur rappelle notamment la publication récente de l'ouvrage que Mauvillon avait fait imprimer quelques années auparavant à Amsterdam. Mais, à la différence de Mauvillon, Scheid avait le mérite d'être impartial. En effet, à l'occasion du compte rendu sur Scheid, le journal de Göttingen rappelle encore une fois les principaux défauts du livre de Mauvillon. Malgré ses mérites, cet auteur se voit accusé de partialité et mis au nombre des auteurs hostiles au Saint-Empire en général et à la maison d'Autriche en particulier, tels que Chemnitz et Blondel. Selon le journal de Göttingen, les positions prises par Mauvillon étaient incompatibles avec la pensée d'un patriote allemand. En revanche, Scheid est considéré comme un auteur modeste et honnête, qui évitait toute forme de partialité. Qui plus est, selon ce compte rendu, l'ouvrage de Scheid était supérieur à tous les autres livres français sur le droit constitutionnel du Saint-Empire.

Toutefois, les »Göttingische Gelehrte Anzeigen« admettent que la »Description du gouvernement présent du Corps germanique« de Necker contenait de nombreuses informations utiles et d'une très grande qualité sur le droit public allemand. Mais le journal de Göttigen reproche à Necker de ne pas avoir traité toutes les parties essentielles de la Constitution allemande, d'avoir été beaucoup plus bref que Scheid sur de nombreux points et de ne pas avoir décidé certains problèmes fondamentaux ${ }^{143}$.

Le journal de Göttingen évoque également l'»Histoire de l'Empire« de Jean Heiss. Bien qu'il s'agît d'un ouvrage historique, non d'un traité de droit public, cette œuvre était souvent utilisée par les Français comme un manuel de droit allemand. Cependant, à Göttingen, on estimait que les informations sur la Constitution allemande qu'on trouvait dans le livre de Heiss étaient mauvaises et superficielles. On portait un jugement identique sur ses traductions des lois fondamentales de l'Empire ${ }^{144}$. En annonçant la publication imminente du quatrième volume du »Traité sistématique«, qui devait reproduire la version française de trois lois fondamentales du Saint-Empire, l'auteur du compte rendu s'étonne que, d'une manière générale, les Français se soient donné si peu de peine à faire traduire cor-

$142 \mathrm{Cf}$. Góttingische Zeitungen, année 1751, p. 388.

143 Le journal de Göttingen reproche aussi à Mauvillon de ne pas avoir bien compris tous les aspects du droit public allemand. Cf. ibid., p. 389-390.

$144 »$ Die Reichs=Histoire des Hrn. Heuß hat sonsten zwar auch bey denen Franzosen die Stelle eines Staats=Rechts vertreten sollen. Das Buch ist allzubekannt, als daß wir seiner hier ausfůhrlich gedencken solten, und wer nur ein wenig darein zu sehen sich Mưhe gegeben hat, wird gestehen müssen, daß alles, was das Staats=Recht angehet, von Hrn. Heuß über die massen schlecht und seichte abgehandelt worden, und er auch nicht einmahl geschickt gewesen sey, die dabey mitgehteilte Stücke solcher gestalten zu übersetzen, daß man sich darauf verlassen kônnte«, ibid., p. 390. 
rectement la paix de Westphalie malgré la part importante que la diplomatie française y avait prise ${ }^{145}$. Or, notre analyse de ses traductions a montré que cette critique est beaucoup trop sévère et ne rend pas justice à l'ouvrage de pionnier que Heiss avait entrepris.

En outre, le journal de Göttingen mentionne l'édition française de la capitulation impériale de Charles VII publiée sous le titre de »Remarques instructives« (il $s$ 'agit très certainement du livre de Jean-François Spon) ${ }^{146}$. Mais cet ouvrage était centré sur les capitulations impériales et n'évoquait donc que les matières qui y étaient traitées. De plus, pensait-on à Göttingen, Spon était un auteur partiall47. En effet, après son élection, Charles VII l'avait nommé son secrétaire intime.

Il est vrai que Scheid ne peut pas être accusé de partialité. Il est un érudit plutôt qu'un politique. Cependant, cette conclusion ne doit pas se fonder sur le compte rendu publié à Göttingen, mais sur la lecture de son ouvrage. En effet, étant donné que le »Traité sistématique «était dédié à l'électeur de Hanovre et roi d'Angleterre, qui était en même temps le protecteur de l'académie de Göttingen, les »Göttingische Gelehrte Anzeigen « avaient tout intérêt à déclarer Scheid impartial, bien que l'identité de l'auteur leur échappât ${ }^{148}$.

Malgré cette réserve, le jugement général que le journal de Göttingen portait sur l'œuvre de Scheid ne nous paraît pas infondé. En effet, l'auteur du compte rendu estimait que cet ouvrage était le premier livre français sur le droit public allemand qui sût convaincre les lecteurs par son caractère exhaustif et par sa précision $^{149}$. Ajoutons, pour notre part, qu'il était peut-être trop long et trop détaillé pour une partie du public français. En outre, il faut également avouer que si, à notre avis, Scheid donna effectivement au public le meilleur ouvrage français sur le

145 "Wer sich die Múhe geben mag, die bisher hievon vorhanden gewesenen franzósischen Uebersezungen mit dem Original zu vergleichen, wird finden, daß dieses keine vergebliche Arbeit seye [c'est-à-dire de traduire en français la capitulation impériale de François Ier, la Bulle d'or et la paix de Westphalie]; und es ist besonders zu verwundern, wie die Herren Franzosen, die doch bey dem Westphâlischen Frieden ein großes Interesse haben, und sonsten so gerne in alle Teutsche Staats=Sachen ihre Hănde schlagen mo̊gen, sich in so langen Jahren nicht einmahl haben einfallen lassen, auf eine rechte Uebersezung desselben bedacht zu seyn«, ibid., p. 391-392.

146 [SPON], La Capitulation de l'Empereur Charles VII.

147 Cf. Góttingische Zeitungen, année 1751, p. 390.

148 En revanche, quand la troisième partie du compte rendu fut publiée, Scheid était connu comme auteur du »Traité sistématique « dans la rédaction des "Gôttingische Gelehrte Anzeigen«, puisque Moser avait publié son nom; mais comme l'auteur ne voulait toujours pas être nommé, la rédaction s'abstint de le citer, cf. Góttingische Anzeigen, année 1753, t. I, p. 131132. Abstraction faite de cette identification et de l'excellente qualité typographique du »Traité sistématique«, le compte rendu de Moser n'apporte pas d'élément intéressant, cf. MOSER, Teutsches Staats=Archiv, neuvième partie (1751), p. 178.

149 „Nach so bewandten Umstănden nun bleibet dem gelehrten Hrn. Verfasser [sc. Scheid] dieses gegenwártigen Wercks das wohlverdiente Lob, daB er noch immer der erste seye, der in Franzósischer Sprache von dem Teutschen Staats=Recht ausfưhrlich und gründlich gehandelt habe, und wir vermuthen, diese seine Abhandlung werde mit einem allgemeinen Beyfall von unpartheyischen Kennern aufgenommen werden «, Goottingische Zeitungen, année 1751, p. 390. 
droit constitutionnel allemand, les Français ne manquaient pas alors de très bons livres sur cette matière.

Le journal de Göttingen reconnâit à Scheid le mérite d'avoir utilisé les bonnes sources et loue l'objectivité de l'auteur quand il traite des litiges juridiques qu'un érudit privé ne doit pas trancher. Dans la première partie du compte rendu, on fait seulement deux reproches à Scheid: d'abord, son plan, fondé sur les »Notitia Sacri Romani Imperii« de Boecler, est critiqué à plusieurs reprises, puisque toutes les matières ne sont pas traitées dans les chapitres appropriés ${ }^{150}$; puis, son exposé d'un litige confessionnel qui avait lieu dans le pays de Hohenlohe est accusé de ne pas être assez précis ${ }^{151}$.

À la différence du premier volume, le tome II du »Traité sistématique« donnait l'occasion à un plus grand nombre de critiques. Mais la plupart des reproches qu'on faisait à Scheid ne concernaient que des précisions ou des adjonctions qu'il aurait pu insérer dans ses chapitres sur l'histoire des différentes maisons électorales qui existaient en Allemagne. En réalité, on pouvait reprocher seulement trois erreurs à Scheid: les »Göttingische Gelehrte Anzeigen« précisaient que, depuis l'époque de l'empereur Otton le Grand, l'archevêque de Mayence n'avait pas toujours conservé le titre d'»archichancelier de l'Empire«; que le titre d'»Archicancellarius per Galliam《 que portait l'électeur de Trèves ne se référait pas à la France entière, mais seulement à la »Gallia Belgica«; et que, selon certaines sources, l'archevêque de Cologne avait déjà porté le titre d'»archichancelier en Italie « avant le règne de Frédéric Ier152.

À Göttingen, on considérait parfois comme trop long le troisième volume du »Traité sistématique«, en particulier les informations historiques que Scheid donnait sur certains droits qui étaient passés hors d'usage au XVIIIe siècle et dont l'évocation ne paraissait donc plus nécessaire dans un traité de droit public. De plus, l'auteur du compte rendu pensait que Scheid n'avait pas suffisamment éclairci les droits du pape sur l'Église catholique allemande; mais il ajoutait qu' aucun ouvrage de droit public n' avait encore traité cette matière d'une manière convaincante. Enfin, on reconnaissait à Scheid le mérite d'avoir consulté beaucoup d'ouvrages récents, d'avoir bien expliqué la dernière capitulation impériale

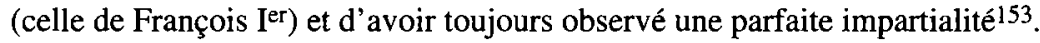

En somme, pour les "Göttingische Gelehrte Anzeigen «, l'œuvre de Scheid était le premier bon livre français sur la Constitution de l'Empire. Le compte rendu du volume II dit explicitement que cet ouvrage méritait la première place (»die Oberstelle«) parmi les livres français sur le droit d'État allemand, et rappelle que le premier volume avait reçu les applaudissements des ministres d'État et des per-

150 Cf. ibid., p. 391-393. Même critique dans les Gôttingische Anzeigen, année 1753, t. I, p. 135.

151 Cf. Gôttingische Zeitungen, année 1751, p. 393-395, en particulier p. 394.

152 Cf. ibid., année 1752, t. I, p. 304-305.

153 Cf. Gôttingische Anzeigen, année 1753, t. I, p. 131-135, en particulier p. 132-135. 
sonnes les plus illustres ${ }^{154}$. En comparant cette recension au compte rendu de l'ouvrage de Mauvillon ${ }^{155}$, on s'aperçoit qu'il ne s'agit pas d'une louange de circonstance. En effet, dans ce compte rendu publié deux ans avant celui du premier volume du »Traité sistématique«, l'auteur prétendait que, à son avis, il n'y avait pas encore de livre vraiment convaincant sur cette matière. Il est donc vrai qu'à Göttingen on attendait, autour de 1750, la publication d'un ouvrage en langue française ayant les qualités du travail de Scheid ${ }^{156}$.

Malgré les avantages de son »Droit public germanique«, Mauvillon ne pouvait pas convaincre les esprits critiques de Göttingen. En effet, on voit que la lecture des premières pages de ce livre avait déjà agacé l'auteur du compte rendu. Mauvillon prétendait qu'au lieu des trois mille ou quatre mille ouvrages qui existaient sur le droit public allemand, il était suffisant d'en lire un seul pour se faire une idée juste de la Constitution de l'Empire. Il se moquait également du langage spécifique des juristes allemands, incompréhensible pour le commun des mortels. Le journal de Göttingen ne commentait même pas ces propos, estimant que le fait de les rapporter suffisait à désavouer l'auteur du $»$ Droit public germanique«. En revanche, à propos de l'assertion de Mauvillon selon laquelle tout le droit public allemand était fondé exclusivement sur la Bulle d'or, la paix de Westphalie et la dernière capitulation impériale, en l'occurrence celle de François Ier, on notait à Göttingen, avec une sévérité mal cachée, que Mauvillon semblait ne jamais avoir lu les autres lois fondamentales du Saint-Empire ${ }^{157}$. En outre, le journal de Göttingen reprochait à cet auteur de ne point parler, dans son chapitre sur l'empereur, des droits exclusifs de celui-ci (les iura reservata impériaux), mais d'énumérer à leur place toutes les choses qu'il n'appartenait pas à l'empereur de faire. Le compte rendu relève des lacunes dans d'autres chapitres, critiquant surtout l'absence d'une énumération précise des princes de l'Empire ecclésiastiques et séculiers.

À Göttingen, on concluait que cet ouvrage ne pouvait être considéré comme un véritable traité du droit public allemand. Cependant, on saluait chez Mauvillon son objectivité dans les affaires de religion, et on notait surtout qu'en général les auteurs français avaient l'habitude de parler du pouvoir papal avec une véracité absolue ("mit einer vo̊lligen Wahrheits=Liebe«). De plus, on estimait que Mauvillon avait fait plus de progrès dans la connaissance du droit public allemand que

154 Gôttingische Zeitungen, année 1752, t. I, p. 303. Jugement identique dans Gôttingische Anzeigen, année 1753, t. I, p. 134-135.

155 Gốttingische Zeitungen, année 1749, p. 905-908.

156 À cause de son caractère incomplet, l'ouvrage de Mauvillon ne pouvait pas combler cette lacune, cf. ibid., p. 907-908: »Unsere geneigte Leser werden nunmehro aus diesem Auszug von selbst urtheilen, wie wenig sie hier ein vollståndiges Teutsches Staats=Recht zu suchen haben, und wie noch immer bey so vielen schónen und guten Frantzósischen Búchern, eine wohlgerathene Abhandlung von dem Teutschen Staats=Recht ein Buch bleibet, welches man vergeblich in dieser Sprache zu lesen wúnschet«.

157 Cf. ibid., p. 906 et 908 (citation): »wir glauben aber doch, daß wir uns nicht irren, wann wir sagen, er [sc. Mauvillon] selber habe die Teutsche Reichs $=$ Grundgeseze ausser denen dreyen, davon der andre Theil handelt, niemalen gelesen «. 
certains de ses compatriotes qui avaient écrit sur le même sujet avant lui. Par contre, on n' appréciait point que Mauvillon considérât l'Empire d'Allemagne comme une partie de l'ancienne monarchie française et qu'il accusât les Allemands d'avoir ravi aux Français la dignité impériale de Charlemagne ${ }^{158}$.

\section{Conclusion}

Pour bien comprendre la valeur des comptes rendus publiés à Göttingen, qui paraissent parfois pointilleux, il faut se rappeler que son université était alors la première école historique et juridique de l'Allemagne. Les Français en étaient conscients. En 1755, les »Mémoires de Trévoux « chantaient les louanges de Schmauss, qui était professeur à Göttingen et dont le »Tableau du gouvernement actuel de l'Empire « venait d'être traduit en français. Consécration s'il en est pour un auteur comme Scheid que d'obtenir une critique aussi favorable de la part des meilleurs juristes de l'Empire dans les »Göttingische Gelehrte Anzeigen«! Or, si Scheid était un auteur allemand, il profita du travail accompli par les auteurs français qui avaient écrit avant lui, et avec lesquels il entretenait parfois des relations personnelles (comme, par exemple, avec Joseph Barre). C'est donc l'aboutissement d'une réflexion séculaire des auteurs francophones sur la Constitution allemande qui est ainsi consacré par les critiques de Göttingen. Selon Moser, Gérard de Rayneval ravit en 1766 la palme du meilleur livre français sur le droit public allemand à Jean-Frédéric Scheid. 